\title{
A Simple Method to Generate Integer Sequences
}

\author{
Kai Wang \\ Henderson, NV, USA \\ Email: kaiwang1945@gmail.com
}

How to cite this paper: Wang, K. (2019) A Simple Method to Generate Integer Sequences. Open Access Library Journal, 6: e5502.

https://doi.org/10.4236/oalib.1105502

Received: May 27, 2019

Accepted: June 18, 2019

Published: June 21, 2019

Copyright $\odot 2019$ by author(s) and Open Access Library Inc.

This work is licensed under the Creative Commons Attribution International License (CC BY 4.0).

http://creativecommons.org/licenses/by/4.0/

\begin{abstract}
We will use a simple method to generate integer sequences whose terms are sums of mixed powers of trigonometric values at angles of a heptagonal triangle. Our results include many new integer sequences and some sequences which are discovered using different methods.
\end{abstract}

\section{Subject Areas}

Mathematical Analysis

\section{Keywords}

Integer Sequences, Mixed Power Sums of Trigonometric Functions

\section{Introduction}

An order- $d$ homogeneous linear recurrence with constant coefficients is an equation of the form

$$
a_{n}=c_{1} a_{n-1}+c_{2} a_{n-2}+\cdots+c_{d} a_{n-d},
$$

where the $d$ coefficients $c_{i}$ (for all $i$ ) are constants, and $c_{d} \neq 0$.

A constant-recursive sequence is a sequence satisfying a recurrence of this form. There are $\mathrm{d}$ degrees of freedom for solutions to this recurrence, i.e., the initial values $a_{0}, \cdots, a_{d-1}$ can be taken to be any values but then the recurrence determines the sequence uniquely.

The same coefficients yield the characteristic polynomial (also "auxiliary polynomial")

$$
p(t)=t^{d}-c_{1} t^{d-1}-c_{2} t^{d-2}-\cdots-c_{d}
$$

whose $d$ roots play a crucial role in finding and understanding the sequences satisfying the recurrence. The following result is from [1]. 
Theorem 1. If the roots $r_{1}, r_{2}, \cdots$ are all distinct, then each solution to the recurrence takes the form

$$
a_{n}=k_{1} r_{1}^{n}+k_{2} r_{2}^{n}+\cdots+k_{d} r_{d}^{n},
$$

where the coefficients $k_{i}$ are determined in order to fit the initial conditions of the recurrence.

The purpose of this paper is to use a simple version of Theorem 1 to generate integer sequences.

Corollary 2. Let $\{X, Y, Z\}$ be the roots of a cubic equation:

$$
t^{3}+a t^{2}+b t+c=0
$$

where $a, b, c$ are integers. Let $\{u, v, w\}$ be three numbers and let

$$
\left\{p(n)=u X^{n}+v Y^{n}+w Z^{n} \mid n=0,1,2, \cdots\right\} .
$$

If $p(0), p(1), p(2)$ are integers then

$$
\{p(n) \mid n=0,1,2, \cdots\}
$$

is an integer sequence with the recurrence relation:

$$
p(n)=-a p(n-1)-b p(n-2)-c p(n-3) .
$$

Integer sequences whose terms are mixed power sums of trigonometric functions at heptagonal triangles have been studied by many researchers such as [2]-[7]. OEIS [2] the On-Line Encyclopedia of Integer Sequences is an online database of integer sequences. It consists of all known integer sequence and related information. It may have recurrence relation, initial values, generating function, references, etc. In Section 21, we include all the known sequences which are based on trigonometric values at heptagonal triangles. R. Witula and his coworkers [4] [5] [6] [7] have developed very difficult and complicate methods to produce integer sequences. In our previous paper [3] we begin using simpler and more direct method to generate sequence. This paper is an extension of that paper. This paper will use above mentioned simple method to generate integer sequences whose terms are sums of mixed powers of trigonometric values at angles of a heptagonal triangle. Our results include many new integer sequences and some sequences which are discovered using different methods.

Note that in this paper, all the sequences and tables are generated by computer.

\section{Sums of Mixed Powers of Trigonometric Functions}

For convenience, in this paper, let $\theta=\frac{\pi}{7}$. We will use many results from [3]. For the convenience of readers, we include them here.

Definition 3. Let $f(2 \theta, 4 \theta, 8 \theta)$ be an expression of trigonometric values in terms of $\{2 \theta, 4 \theta, 8 \theta\}$. Let

$$
\sum f(2 \theta, 4 \theta, 8 \theta)=f(2 \theta, 4 \theta, 8 \theta)+f(4 \theta, 8 \theta, 2 \theta)+f(8 \theta, 2 \theta, 4 \theta) .
$$


Definition 4. For an integer $n$, let

$$
\begin{aligned}
& S(n)=\sum \sin ^{n}(2 \theta)=\sin ^{n}(2 \theta)+\sin ^{n}(4 \theta)+\sin ^{n}(8 \theta), \\
& C(n)=\sum \cos ^{n}(2 \theta)=\cos ^{n}(2 \theta)+\cos ^{n}(4 \theta)+\cos ^{n}(8 \theta), \\
& T(n)=\sum \tan ^{n}(2 \theta)=\tan ^{n}(2 \theta)+\tan ^{n}(4 \theta)+\tan ^{n}(8 \theta) .
\end{aligned}
$$

For integers, $m, n$, let

$$
\begin{aligned}
& W(m, n)=\sum \sin ^{m}(2 \theta) \sin ^{n}(4 \theta) \\
& =\sin ^{m}(2 \theta) \sin ^{n}(4 \theta)+\sin ^{m}(4 \theta) \sin ^{n}(8 \theta)+\sin ^{m}(8 \theta) \sin ^{n}(2 \theta) .
\end{aligned}
$$

Let

$$
P=\sin (2 \theta) \sin (4 \theta) \sin (8 \theta) .
$$

We start with the following results which can be proved easily from trigonometric identities from [8] [9].

Proposition 5. Let $S(n)$ and $P$ be defined as in Definition 4.

1) The values $\{\sin (2 \theta), \sin (4 \theta), \sin (8 \theta)\}$ are the roots of the equation

$$
x^{3}-\frac{\sqrt{7}}{2} x^{2}+\frac{\sqrt{7}}{8}=0 \text {. }
$$

2) $P=-\frac{\sqrt{7}}{8}$

3) For an integer $n, S(n)$ satisfies the recurrence relations:

$$
\begin{gathered}
S(n)=\frac{\sqrt{7}}{2} S(n-1)-\frac{\sqrt{7}}{8} S(n-3), \\
S(-n)=4 S(-n+2)-\frac{8 \sqrt{7}}{7} S(-n+3) .
\end{gathered}
$$

Using this recurrence relation we can compute $S(n)$ for any integer $n$. In the following, we will only show a few terms which will be used in later applications.

Proposition 6. With above notations, the values of $S(n)$ for $n=0, \cdots, \pm 19$ are as follows.

\begin{tabular}{cccccc}
\hline$n$ & 0 & 1 & 2 & 3 & 4 \\
$S(n)$ & 3 & $\frac{\sqrt{7}}{2}$ & $\frac{7}{2^{2}}$ & $\frac{\sqrt{7}}{2}$ & $\frac{3 \times 7}{2^{4}}$ \\
$S(-n)$ & 3 & 0 & $2^{3}$ & $-\frac{3 \cdot 2^{3} \sqrt{7}}{7}$ & $2^{5}$ \\
\hline$n$ & 5 & 6 & 7 & 8 & 9 \\
$S(n)$ & $\frac{7 \sqrt{7}}{2^{4}}$ & $\frac{5 \times 7}{2^{5}}$ & $\frac{7^{2} \sqrt{7}}{2^{7}}$ & $\frac{5 \times 7^{2}}{2^{8}}$ & $\frac{25 \times 7 \sqrt{7}}{2^{9}}$ \\
$S(-n)$ & $-\frac{5 \times 2^{5} \sqrt{7}}{7}$ & $\frac{17 \times 2^{6}}{7}$ & $-2^{7} \sqrt{7}$ & $\frac{11 \times 2^{9}}{7}$ & $-\frac{33 \times 2^{10} \sqrt{7}}{7^{2}}$ \\
\hline$n$ & 10 & 11 & 12 & $\frac{13}{2^{2}}$ & 14 \\
$S(n)$ & $\frac{9 \times 7^{2}}{2^{9}}$ & $\frac{13 \times 7^{2} \sqrt{7}}{2^{11}}$ & $\frac{33 \times 7^{2}}{2^{11}}$ & $\frac{3 \times 7^{2} \sqrt{7}}{2^{9}}$ & $\frac{5 \times 7^{4}}{2^{14}}$ \\
\hline
\end{tabular}




\section{Continued}

\begin{tabular}{cccccc}
\hline$S(-n)$ & $\frac{29 \times 2^{10}}{7}$ & $-\frac{11 \times 2^{14} \sqrt{7}}{7^{2}}$ & $\frac{269 \times 2^{12}}{7^{2}}$ & $-\frac{117 \times 2^{13} \sqrt{7}}{7^{2}}$ & $\frac{51 \times 2^{14}}{7}$ \\
\hline$n$ & 15 & 16 & 17 & 18 & 19 \\
$S(n)$ & $\frac{179 \times 7^{2} \sqrt{7}}{2^{15}}$ & $\frac{131 \times 7^{3}}{2^{16}}$ & $\frac{3 \times 7^{3} \sqrt{7}}{2^{12}}$ & $\frac{493 \times 7^{3}}{2^{18}}$ & $\frac{181 \times 7^{3} \sqrt{7}}{2^{18}}$ \\
$S(-n)$ & $-\frac{17 \times 2^{21} \sqrt{7}}{7^{3}}$ & $\frac{237 \times 2^{17}}{7^{2}}$ & $-\frac{1445 \times 2^{17} \sqrt{7}}{7^{3}}$ & $\frac{2203 \times 2^{19}}{7^{3}}$ & $-\frac{1919 \times 2^{19} \sqrt{7}}{7^{3}}$ \\
\hline
\end{tabular}

Proposition 7. Let $C(n)$ be defined as in Definition 4 .

1) The values $\{\cos (2 \theta), \cos (4 \theta), \cos (8 \theta)\}$ are the roots of the equation

$$
x^{3}+\frac{1}{2} x^{2}-\frac{1}{2} x-\frac{1}{8}=0 .
$$

2) $C(n)$ satisfy the following recurrence relations:

$$
\begin{aligned}
C(n) & =-\frac{1}{2} C(n-1)+\frac{1}{2} C(n-2)+\frac{1}{8} C(n-3), \\
C(-n) & =-4 C(-n+1)+4 C(-n+2)+8 C(-n+3) .
\end{aligned}
$$

Using this recurrence relation we can compute $C(n)$ for any integer $n$. In the following, we will only show a few terms which will be used in later applications.

Proposition 8. With above notations, the values of $C(n)$ for $n=0, \cdots, 19$ are as follows.

\begin{tabular}{cccccc}
\hline$n$ & 0 & 1 & 2 & 3 & 4 \\
$C(n)$ & 3 & $-\frac{1}{2}$ & $\frac{5}{2^{2}}$ & $-\frac{1}{2}$ & $\frac{13}{2^{4}}$ \\
$C(-n)$ & 3 & $-2^{2}$ & $3 \times 2^{3}$ & $-11 \times 2^{3}$ & $13 \times 2^{5}$ \\
\hline$n$ & 5 & 6 & 7 & 8 & 9 \\
$C(n)$ & $-\frac{1}{2}$ & $\frac{19}{2^{5}}$ & $-\frac{57}{2^{7}}$ & $\frac{117}{2^{8}}$ & $-\frac{193}{2^{9}}$ \\
$C(-n)$ & $-57 \times 2^{5}$ & $129 \times 2^{6}$ & $-289 \times 2^{7}$ & $325 \times 2^{9}$ & $-365 \times 2^{11}$ \\
\hline$n$ & 10 & 11 & 12 & 13 & 14 \\
$C(n)$ & $\frac{185}{2^{9}}$ & $-\frac{639}{2^{11}}$ & $\frac{593}{2^{11}}$ & $-\frac{1047}{2^{12}}$ & $\frac{3827}{2^{14}}$ \\
$C(-n)$ & $3281 \times 2^{10}$ & $-1843 \times 2^{13}$ & $16565 \times 2^{12}$ & $-37221 \times 2^{13}$ & $83635 \times 2^{14}$ \\
\hline$n$ & 15 & 16 & 17 & 18 & 19 \\
$C(n)$ & $-\frac{6829}{2^{15}}$ & $\frac{12389}{2^{16}}$ & $-\frac{5555}{2^{15}}$ & $\frac{40169}{2^{18}}$ & $-\frac{18055}{2^{17}}$ \\
$C(-n)$ & $-93963 \times 2^{16}$ & $211133 \times 2^{17}$ & $-948823 \times 2^{17}$ & $1065993 \times 2^{19}$ & $-4790529 \times 2^{19}$ \\
\hline
\end{tabular}

Proposition 9. Let $T(n)$ be defined as in Definition 4.

1) The values $\{\tan (2 \theta), \tan (4 \theta), \tan (8 \theta)\}$ are the roots of the equation 


$$
x^{3}+\sqrt{7} x^{2}-7 x+\sqrt{7}=0 .
$$

2) $T(n)$ satisfy the following recurrence relations.

$$
\begin{gathered}
T(n)=-\sqrt{7} T(n-1)+7 T(n-2)-\sqrt{7} T(n-3), \\
T(-n)=\sqrt{7} T(-n+1)-T(-n+2)-\frac{\sqrt{7}}{7} T(-n+3) .
\end{gathered}
$$

Using this recurrence relation we can compute $T(n)$ for any integer $n$. In the following, we will only show a few terms which will be used in later applications and for future reference.

\begin{tabular}{|c|c|c|c|c|c|}
\hline$n$ & 0 & 1 & 2 & 3 & 4 \\
\hline$T(n)$ & 3 & $-\sqrt{7}$ & $3 \times 7$ & $-31 \sqrt{7}$ & $53 \times 7$ \\
\hline$T(-n)$ & 3 & $\sqrt{7}$ & 5 & $\frac{25 \sqrt{7}}{7}$ & 19 \\
\hline$n$ & 5 & 6 & 7 & 8 & 9 \\
\hline$T(n)$ & $-87 \times 7 \sqrt{7}$ & $1011 \times 7$ & $-239 \times 7^{2} \sqrt{7}$ & $2771 \times 7^{2}$ & $-\frac{32119 \times 7 \sqrt{7}}{1}$ \\
\hline$T(-n)$ & $\frac{103 \sqrt{7}}{7}$ & $\frac{563}{7}$ & $9 \times 7 \sqrt{7}$ & $\frac{2421}{7}$ & $\frac{13297 \sqrt{7}}{7^{2}}$ \\
\hline$n$ & 10 & 11 & 12 & 13 & 14 \\
\hline$T(n)$ & $53189 \times 7^{2}$ & $-88079 \times 7^{2} \sqrt{7}$ & $1020995 \times 7^{2}$ & $-1690737 \times 7^{2} \sqrt{7}$ & $57139 \times 7^{5}$ \\
\hline$T(-n)$ & $\frac{10435}{7}$ & $\frac{57327 \sqrt{7}}{7^{2}}$ & $\frac{314947}{7^{2}}$ & $\frac{247185 \sqrt{7}}{7^{2}}$ & $\frac{194003}{7}$ \\
\hline$n$ & 15 & 16 & 17 & 18 & 19 \\
\hline$T(n)$ & $-32454831 \times 7^{2} \sqrt{7}$ & $53744245 \times 7^{3}$ & $-88998887 \times 7^{3} \sqrt{7}$ & $1031656755 \times 7^{3}$ & $-1708393209 \times 7^{3} \sqrt{7}$ \\
\hline$T(-n)$ & $\frac{7460905 \sqrt{7}}{7^{3}}$ & $\frac{5855699}{7^{2}}$ & $\frac{32170967 \sqrt{7}}{7^{3}}$ & $\frac{176745971}{7^{3}}$ & $\frac{138719305 \sqrt{7}}{7^{3}}$ \\
\hline
\end{tabular}

Proposition 10. With above notations, the values of $T(n)$ for $n=0, \cdots, 19$ are as follows.

Proposition 11. Let $W(i, j)$ be defined as in Definition 4. Then $W(i, j)$ satisfy the following recurrence relations.

$$
W(i, j)=\frac{\sqrt{7}}{2} W(i-1, j)-\frac{\sqrt{7}}{8} W(i-3, j),
$$

and

$$
W(i, j)=\frac{\sqrt{7}}{2} W(i, j-1)-\frac{\sqrt{7}}{8} W(i, j-3) .
$$

Proof. The recurrence relations follow easily from the recurrence relations in Proposition 5.

Remark 12.

1) For an integer $n, W(0, n)=W(n, 0)=S(n)$.

2) We only need the values $W(m, n)$ for $m, n \geq 0$.

Proposition 13. With the above notations, list of 


\begin{tabular}{cccccccc}
\hline$m \backslash n$ & 1 & 2 & 3 & 4 & 5 & 6 & 7 \\
1 & 0 & $\frac{\sqrt{7}}{2^{2}}$ & $\frac{7}{2^{4}}$ & $\frac{7 \sqrt{7}}{2^{5}}$ & $\frac{5 \times 7}{2^{6}}$ & $\frac{7 \sqrt{7}}{2^{5}}$ & $\frac{3 \times 7^{2}}{2^{8}}$ \\
2 & $\frac{\sqrt{7}}{2^{3}}$ & $\frac{7}{2^{3}}$ & $\frac{7 \sqrt{7}}{2^{5}}$ & $\frac{3 \times 7}{2^{5}}$ & $\frac{7 \sqrt{7}}{2^{5}}$ & $\frac{3 \times 7^{2}}{2^{8}}$ & $\frac{15 \times 7 \sqrt{7}}{2^{9}}$ \\
3 & 0 & $\frac{7 \sqrt{7}}{2^{5}}$ & $\frac{3 \times 7}{2^{6}}$ & $\frac{3 \times 7 \sqrt{7}}{2^{7}}$ & $\frac{7^{2}}{2^{7}}$ & $\frac{11 \times 7 \sqrt{7}}{2^{9}}$ & $\frac{7^{2}}{2^{7}}$ \\
4 & 0 & $\frac{5 \times 7}{2^{6}}$ & $\frac{7 \sqrt{7}}{2^{6}}$ & $\frac{7^{2}}{2^{7}}$ & $\frac{9 \times 7 \sqrt{7}}{2^{9}}$ & $\frac{7^{3}}{2^{10}}$ & $\frac{5 \times 7^{2} \sqrt{7}}{2^{11}}$ \\
5 & $-\frac{7}{2^{6}}$ & $\frac{3 \times 7 \sqrt{7}}{2^{7}}$ & $\frac{7^{2}}{2^{8}}$ & $\frac{7 \sqrt{7}}{2^{6}}$ & $\frac{5 \times 7^{2}}{2^{10}}$ & $\frac{7^{2} \sqrt{7}}{2^{9}}$ & $\frac{5 \times 7^{2}}{2^{10}}$ \\
6 & $-\frac{7 \sqrt{7}}{2^{7}}$ & $\frac{7^{2}}{2^{7}}$ & $\frac{7 \sqrt{7}}{2^{7}}$ & $\frac{5 \times 7^{2}}{2^{10}}$ & $\frac{3 \times 7^{2} \sqrt{7}}{2^{11}}$ & $\frac{17 \times 7^{2}}{2^{12}}$ & $\frac{3 \times 7^{2} \sqrt{7}}{2^{11}}$ \\
7 & $-\frac{7^{2}}{2^{8}}$ & $\frac{9 \times 7 \sqrt{7}}{2^{9}}$ & $\frac{7^{2}}{2^{9}}$ & $\frac{3 \times 7^{2} \sqrt{7}}{2^{11}}$ & $\frac{3 \times 7^{2}}{2^{10}}$ & $\frac{5 \times 7^{2} \sqrt{7}}{2^{12}}$ & $\frac{7^{4}}{2^{14}}$
\end{tabular}

$8 \quad-\frac{3 \times 7 \sqrt{7}}{2^{8}} \quad \frac{3 \times 7^{2}}{2^{9}} \quad \frac{7^{2} \sqrt{7}}{2^{11}} \quad \frac{13 \times 7^{2}}{2^{12}} \quad \frac{7^{3} \sqrt{7}}{2^{13}} \quad \frac{3 \times 7^{3}}{2^{13}} \quad \frac{29 \times 7^{2} \sqrt{7}}{2^{15}}$

$9 \quad-\frac{5 \times 7^{2}}{2^{10}} \quad \frac{7^{2} \sqrt{7}}{2^{9}} \quad \frac{3 \times 7^{2}}{2^{12}} \quad \frac{7^{2} \sqrt{7}}{2^{10}} \quad \frac{7^{3}}{2^{12}} \quad \frac{25 \times 7^{2} \sqrt{7}}{2^{15}} \quad \frac{17 \times 7^{3}}{2^{16}}$

$\begin{array}{cccccccc}10 & -\frac{7^{2} \sqrt{7}}{2^{9}} & \frac{19 \times 7^{2}}{2^{12}} & \frac{7^{2} \sqrt{7}}{2^{13}} & \frac{5 \times 7^{3}}{2^{1^{4}}} & \frac{7^{2} \sqrt{7}}{2^{11}} & \frac{15 \times 7^{3}}{2^{16}} & \frac{5 \times 7^{3} \sqrt{7}}{2^{16}} \\ m \backslash n & 8 & 9 & 10 & 11 & 12 & 13 & 14\end{array}$

$1 \quad \frac{7 \sqrt{7}}{2^{5}} \quad \frac{3 \times 7^{2}}{2^{8}} \quad \frac{9 \times 7^{2} \sqrt{7}}{2^{11}} \quad \frac{47 \times 7^{2}}{2^{12}} \quad \frac{5 \times 7^{3} \sqrt{7}}{2^{13}} \quad \frac{13 \times 7^{3}}{2^{13}} \quad \frac{135 \times 7^{2} \sqrt{7}}{2^{15}}$

$2 \quad \frac{11 \times 7^{2}}{2^{10}} \quad \frac{7^{2} \sqrt{7}}{2^{8}} \quad \frac{41 \times 7^{2}}{2^{12}} \quad \frac{15 \times 7^{2} \sqrt{7}}{2^{12}} \quad \frac{11 \times 7^{3}}{2^{13}} \quad \frac{113 \times 7^{2} \sqrt{7}}{2^{15}} \quad \frac{83 \times 7^{3}}{2^{16}}$

$3 \quad \frac{3 \times 7^{2} \sqrt{7}}{2^{10}} \quad \frac{31 \times 7^{2}}{2^{12}} \quad \frac{23 \times 7^{2} \sqrt{7}}{2^{13}} \quad \frac{17 \times 7^{3}}{2^{14}} \quad \frac{11 \times 7^{2} \sqrt{7}}{2^{12}} \quad \frac{65 \times 7^{3}}{2^{16}} \quad \frac{3 \times 7^{3} \sqrt{7}}{2^{13}}$

$4 \quad \frac{13 \times 7^{2}}{2^{11}} \quad \frac{19 \times 7^{2} \sqrt{7}}{2^{13}} \quad \frac{7^{4}}{2^{13}} \quad \frac{9 \times 7^{2} \sqrt{7}}{2^{12}} \quad \frac{53 \times 7^{3}}{2^{16}} \quad \frac{39 \times 7^{3} \sqrt{7}}{2^{17}} \quad \frac{201 \times 7^{3}}{2^{18}}$

$5 \frac{15 \times 7^{2} \sqrt{7}}{2^{13}} \quad \frac{11 \times 7^{3}}{2^{14}} \quad \frac{57 \times 7^{2} \sqrt{7}}{2^{15}} \quad \frac{3 \times 7^{4}}{2^{15}} \quad \frac{31 \times 7^{3} \sqrt{7}}{2^{17}} \quad \frac{5 \times 7^{3}}{2^{13}} \quad \frac{59 \times 7^{3} \sqrt{7}}{2^{18}}$

$6 \quad \frac{9 \times 7^{3}}{2^{14}} \quad \frac{23 \times 7^{2} \sqrt{7}}{2^{14}} \quad \frac{17 \times 7^{3}}{2^{15}} \quad \frac{25 \times 7^{3} \sqrt{7}}{2^{17}} \quad \frac{129 \times 7^{3}}{2^{18}} \quad \frac{95 \times 7^{3} \sqrt{7}}{2^{19}} \quad \frac{5 \times 7^{5}}{2^{19}}$

$7 \quad \frac{37 \times 7^{2} \sqrt{7}}{2^{15}} \quad \frac{27 \times 7^{3}}{2^{16}} \quad \frac{5 \times 7^{3} \sqrt{7}}{2^{15}} \quad \frac{103 \times 7^{3}}{2^{18}} \quad \frac{19 \times 7^{3} \sqrt{7}}{2^{17}} \quad \frac{7^{5}}{2^{17}} \quad \frac{289 \times 7^{3} \sqrt{7}}{2^{21}}$

$8 \quad \frac{11 \times 7^{3}}{2^{15}} \quad \frac{7^{3} \sqrt{7}}{2^{13}} \quad \frac{83 \times 7^{3}}{2^{18}} \quad \frac{61 \times 7^{3} \sqrt{7}}{2^{19}} \quad \frac{45 \times 7^{4}}{2^{20}} \quad \frac{29 \times 7^{3} \sqrt{7}}{2^{18}} \quad \frac{171 \times 7^{4}}{2^{22}}$

$9 \frac{13 \times 7^{3} \sqrt{7}}{2^{17}} \quad \frac{33 \times 7^{3}}{2^{17}} \quad \frac{7^{5} \sqrt{7}}{2^{19}} \quad \frac{9 \times 7^{4}}{2^{18}} \quad \frac{93 \times 7^{3} \sqrt{7}}{2^{20}} \quad \frac{137 \times 7^{4}}{2^{22}} \quad \frac{101 \times 7^{4} \sqrt{7}}{2^{23}}$

$10 \quad \frac{27 \times 7^{3}}{2^{17}} \quad \frac{39 \times 7^{3} \sqrt{7}}{2^{19}} \quad \frac{29 \times 7^{4}}{2^{20}} \quad \frac{149 \times 7^{3} \sqrt{7}}{2^{21}} \quad \frac{55 \times 7^{4}}{2^{21}} \quad \frac{81 \times 7^{4} \sqrt{7}}{2^{23}} \quad \frac{209 \times 7^{4}}{2^{23}}$

$$
W(m, m), m=1, \cdots, 10, n=1, \cdots, 14:
$$


Proof.

$$
\begin{gathered}
W(0,0)=3 . \\
W(0,1)=W(1,0)=S(1)=\frac{\sqrt{7}}{2} . \\
W(0,2)=W(2,0)=S(2)=\frac{7}{4} . \\
W(1,1)=\sum \sin (2 \theta) \sin (4 \theta)=P \sum \frac{1}{\sin (8 \theta)}=P S(-1)=0 . \\
W(2,2)=\sum \sin ^{2}(2 \theta) \sin ^{2}(4 \theta)=P^{2} \sum \frac{1}{\sin ^{2}(8 \theta)}=P^{2} S(-2)=\frac{7}{8} . \\
W(1,2)=\sum \sin (2 \theta) \sin ^{2}(4 \theta)=\sum \sin (2 \theta) \sin ^{2}(4 \theta) \cdot \frac{\sin (8 \theta)}{\sin (8 \theta)} \\
=P \sum \frac{\sin (4 \theta)}{\sin (8 \theta)}=P \sum \frac{\sin (4 \theta)}{2 \sin (4 \theta) \cos (4 \theta)} \\
=\frac{P}{2} \sum \frac{1}{\cos (4 \theta)}=\frac{P}{2} C(-1)=\frac{\sqrt{7}}{4} . \\
W(2,1)=\sum \sin ^{2}(2 \theta) \sin (4 \theta)=\sum \sin ^{2}(2 \theta) \sin (4 \theta) \cdot \frac{\sin (8 \theta)}{\sin (8 \theta)} \\
=P \sum \frac{\sin (2 \theta)}{\sin (8 \theta)}=P \sum \frac{\sin (16 \theta)}{\sin (8 \theta)}=P \sum \frac{2 \sin (8 \theta) \cos (8 \theta)}{\sin (8 \theta)} \\
=2 P \sum \cos (8 \theta)=2 P C(1)=\frac{\sqrt{7}}{8} .
\end{gathered}
$$

Then all the other $W(i, j)$ can be computed using recurrence relations in Proposition 5.

\section{List of Support Triads}

In this paper we will use the following list of triads.
1) $S T S 1=\frac{1}{2 \sqrt{7} \sin (2 \theta)}, S T S 2=\frac{1}{2 \sqrt{7} \sin (4 \theta)}, S T S 3=\frac{1}{2 \sqrt{7} \sin (8 \theta)}$.
2) $S T C 1=2 \cos (2 \theta), S T C 2=2 \cos (4 \theta), S T C 3=2 \cos (8 \theta)$.
3) $S T T 1=\frac{1}{\sqrt{7} \tan (2 \theta)}, S T T 2=\frac{1}{\sqrt{7} \tan (4 \theta)}, S T T 3=\frac{1}{\sqrt{7} \tan (8 \theta)}$.

\section{Lemma 14.}

$$
S T S 1+S T S 2+S T S 3=0 .
$$

Proof. By Proposition 5,

$$
\begin{aligned}
& \text { STS } 1+S T S 2+S T S 3 \\
& =\sum \frac{1}{2 \sqrt{7} \sin (2 \theta)} \\
& =\frac{1}{2 \sqrt{7} \sin (2 \theta) \sin (4 \theta) \sin (8 \theta)} \sum \sin (2 \theta) \sin (4 \theta) \\
& =0 .
\end{aligned}
$$




\section{Lemma 15.}

$$
S T C 1+S T C 2+S T C 3=-1 .
$$

Proof. By Proposition 7,

$$
S T C 1+S T C 2+S T C 3=\sum 2 \cos (2 \theta)=2 \sum \cos (2 \theta)=-1 .
$$

\section{Lemma 16.}

$$
S T T 1+S T T 2+S T T 3=1 .
$$

Proof. By Proposition 9,

$$
\begin{aligned}
& S T T 1+S T T 2+S T T 3 \\
& =\sum \frac{1}{\sqrt{7} \tan (2 \theta)} \\
& =\frac{1}{\sqrt{7} \tan (2 \theta) \tan (4 \theta) \tan (8 \theta)} \sum \tan (2 \theta) \tan (4 \theta) \\
& =1 .
\end{aligned}
$$

We will frequently use the following lemma to convert cosine values and tangent values into sine values.

\section{Lemma 17.}

$$
\begin{aligned}
& \cos (x)=\frac{\sin (2 x)}{2 \sin (x)}, \\
& \tan (x)=\frac{2 \sin ^{2}(x)}{\sin (2 x)} .
\end{aligned}
$$

\section{Integer Sequences-S1}

In this section, let

$$
X=4 \sin ^{2}(2 \theta), Y=4 \sin ^{2}(4 \theta), Z=4 \sin ^{2}(8 \theta) .
$$

Proposition 18. With above notations, then $\{X, Y, Z\}$ are the roots of the integer equation

$$
x^{3}-7 x^{2}+14 x-7=0
$$

Proof.

$$
\begin{gathered}
X+Y+Z=\sum 4 \sin ^{2}(2 \theta)=4 S(2)=7 . \\
X Y+Y Z+Z X=\sum\left(4 \sin ^{2}(2 \theta)\right)\left(4 \sin ^{2}(4 \theta)\right)=16 W(2,2)=14 . \\
X Y Z=\left(4 \sin ^{2}(2 \theta)\right)\left(4 \sin ^{2}(4 \theta)\right)\left(4 \sin ^{2}(8 \theta)\right)=64 P^{2}=7 .
\end{gathered}
$$

It follows that $\{X, Y, Z\}$ are the roots of the Equation (4).

Proposition 19. For case
1) $u=1, v=1, w=1$.
2) $u=S T S 1, v=S T S 2, w=S T S 3$.
3) $u=S T S 2, v=S T S 3, w=S T S 1$.
4) $u=S T S 3, v=S T S 1, w=S T S 2$.
5) $u=S T C 1, v=S T C 2, w=S T C 3$. 
6) $u=S T C 2, v=S T C 3, w=S T C 1$.

7) $u=S T C 3, v=S T C 1, w=S T C 2$.

8) $u=S T T 1, v=S T T 2, w=S T T 3$.

9) $u=S T T 2, v=S T T 3, w=S T T 1$.

10) $u=S T T 3, v=S T T 1, w=S T T 2$.

Then

$$
\left\{p(n)=u X^{n}+v Y^{n}+w Z^{n} \mid n=0,1,2, \cdots\right\}
$$

is an integer sequence with the recurrence relation

$$
p(n)=7 p(n-1)-14 p(n-2)+7 p(n-3) .
$$

Proof. We will only prove case $1,4,7,10$. The proofs for other cases are similar. Case 1:

$$
\begin{aligned}
& p(0)=u+v+w=3 . \\
& p(1)=u X+u Y+u Z=7 . \\
p(2)= & u X^{2}+v Y^{2}+w Z^{2} \\
= & (X+Y+Z)^{2}-2(X Y+Y Z+Z X) \\
= & 21 .
\end{aligned}
$$

Case 4: By Lemma 14, $p(0)=0$.

$$
\begin{aligned}
p(1) & =u X+v Y+w Z=\sum\left(\frac{1}{2 \sqrt{7} \sin (8 \theta)}\right)\left(4 \sin ^{2}(2 \theta)\right) \\
& =\frac{2}{P \sqrt{7}} W(3,1)=0 . \\
p(2) & =u X^{2}+v Y^{2}+w Z^{2}=\sum\left(\frac{1}{2 \sqrt{7} \sin (8 \theta)}\right)\left(4 \sin ^{2}(2 \theta)\right)^{2} \\
& =\frac{8}{P \sqrt{7}} W(5,1)=1 .
\end{aligned}
$$

Case 7: By Lemma 15, $p(0)=-1$.

$$
\begin{aligned}
p(1) & =u X+v Y+w Z=\sum(2 \cos (8 \theta))\left(4 \sin ^{2}(2 \theta)\right) \\
& =2 \sum\left(\frac{\sin (16 \theta)}{2 \sin (8 \theta)}\right)\left(4 \sin ^{2}(2 \theta)\right) \\
& =4 \sum \frac{\sin ^{3}(2 \theta)}{\sin (8 \theta)}=\frac{4}{P} W(4,1)=0 . \\
p(2)= & u X^{2}+v Y^{2}+w Z^{2}=\sum(2 \cos (8 \theta))\left(4 \sin ^{2}(2 \theta)\right)^{2} \\
= & 2 \sum\left(\frac{\sin (16 \theta)}{2 \sin (8 \theta)}\right)\left(4 \sin ^{2}(2 \theta)\right)^{2} \\
= & 16 \sum \frac{\sin ^{5}(2 \theta)}{\sin ^{2}(8 \theta)}=\frac{16}{P} W(6,1)=7 .
\end{aligned}
$$

Case 10: By Lemma 16, $p(0)=1$. 


$$
\begin{aligned}
p(1) & =u X+v Y+w Z=\sum\left(\frac{1}{\sqrt{7} \tan (8 \theta)}\right)\left(4 \sin ^{2}(2 \theta)\right) \\
& =\frac{1}{\sqrt{7}} \sum\left(\frac{\sin (16 \theta)}{2 \sin ^{2}(8 \theta)}\right)\left(4 \sin ^{2}(2 \theta)\right) \\
& =\frac{2}{\sqrt{7}} \sum \frac{\sin ^{3}(2 \theta)}{\sin ^{2}(8 \theta)}=\frac{2}{\sqrt{7} P^{2}} W(5,2)=3 . \\
p(2) & =u X^{2}+v Y^{2}+w Z^{2}=\sum\left(\frac{1}{\sqrt{7} \tan (8 \theta)}\right)\left(4 \sin ^{2}(2 \theta)\right)^{2} \\
& =\frac{1}{\sqrt{7}} \sum\left(\frac{\sin (16 \theta)}{2 \sin ^{2}(8 \theta)}\right)\left(4 \sin ^{2}(2 \theta)\right)^{2} \\
& =\frac{8}{\sqrt{7}} \sum \frac{\sin ^{5}(2 \theta)}{\sin ^{2}(8 \theta)}=\frac{8}{\sqrt{7} P^{2}} W(7,2)=9 .
\end{aligned}
$$

Example 20. List of associated sequences:

For case

1) $3,7,21,70,245,882,3234,12005, \cdots$ [Wang A322459]

2) $0,1,4,14,49,175,637,2352,8771,32928, \cdots$ [Witula A215493]

3) $0,-1,-5,-21,-84,-329,-1274,-4900,-18767,-71687, \cdots$ [Witula A215008]

4) $0,0,1,7,35,154,637,2548,9996,38759, \cdots$ [Witula A217274]

5) $-1,0,0,-7,-49,-245,-1078,-4459,-17836,-69972, \cdots$ [Adamson A094430]

6) $-1,-7,-28,-105,-392,-1470,-5537,-20923,-79233,-300468, \cdots$

7) $-1,0,7,42,196,833,3381,13377,52136,201341, \cdots$ [Adamson A094429]

8) $1,1,1,0,-7,-42,-196,-833,-3381,-13377, \cdots$ [Adamson A094429]

9) $1,3,11,42,161,616,2352,8967,34153,129997, \cdots$ [Wang A319512]

10) $1,3,9,28,91,308,1078,3871,14161,52479, \cdots \quad$ [Witula A215007]

\section{Integer Sequences-S2}

In this section, let

$$
X=\frac{\sin (2 \theta)}{\sin (4 \theta)}, Y=\frac{\sin (4 \theta)}{\sin (8 \theta)}, Z=\frac{\sin (8 \theta)}{\sin (2 \theta)} .
$$

Proposition 21. With above notations, $\{X, Y, Z\}$ are the roots of the integer equation

$$
x^{3}+2 x^{2}-x-1=0
$$

Proof.

$$
\begin{gathered}
X+Y+Z=\sum \frac{\sin (2 \theta)}{\sin (4 \theta)}=\frac{1}{P} W(1,2)=-2, \\
X Y+Y Z+Z X=\sum\left(\frac{\sin (2 \theta)}{\sin (4 \theta)}\right)\left(\frac{\sin (4 \theta)}{\sin (8 \theta)}\right)=\frac{1}{P} W(2,1)=-1,
\end{gathered}
$$




$$
X Y Z=\left(\frac{\sin (2 \theta)}{\sin (4 \theta)}\right)\left(\frac{\sin (4 \theta)}{\sin (8 \theta)}\right)\left(\frac{\sin (8 \theta)}{\sin (2 \theta)}\right)=1 .
$$

It follows that $\{X, Y, Z\}$ are the roots of the Equation (5).

Proposition 22. For case
1) $u=1, v=1, w=1$.
2) $u=S T S 1, v=S T S 2, w=S T S 3$.
3) $u=S T S 2, v=S T S 3, w=S T S 1$.
4) $u=S T S 3, v=S T S 1, w=S T S 2$.
5) $u=S T C 1, v=S T C 2, w=S T C 3$.
6) $u=S T C 2, v=S T C 3, w=S T C 1$.
7) $u=S T C 3, v=S T C 1, w=S T C 2$.
8) $u=S T T 1, v=S T T 2, w=S T T 3$.
9) $u=S T T 2, v=S T T 3, w=S T T 1$.
10) $u=S T T 3, v=S T T 1, w=S T T 2$.

Then

$$
\left\{p(n)=u X^{n}+v Y^{n}+w Z^{n} \mid n=0,1,2, \cdots\right\}
$$

is an integer sequence with the recurrence relation

$$
p(n)=-2 p(n-1)+p(n-2)+p(n-3) .
$$

Proof. The proof is similar to the proof of Proposition 19.

Example 23. List of associated sequences:

1) $3,2,6,11,26,57,129, \cdots$ [Wang A322235]

2) $0,0,1,-2,5,-11,25,-56,126,-283, \cdots \quad$ [Sloane A006054]

3) $0,1,-2,5,-11,25,-56,126,-283,636, \cdots$ [Sloane A006054]

4) $0,-1,1,-3,6,-14,31,-70,157,-353, \cdots \quad$ [Sloane A006356, A077998, Bagula $\underline{\text { A106803, }}$ Adamson A180262, Deleham A199853]

5) $-1,3,-2,6,-11,26,-57,129,-289,650, \cdots \quad$ [Sloane A033304, Wang $\underline{\mathrm{A} 274975}$

6) $-1,3,-9,20,-46,103,-232,521,-1171,2631, \cdots \quad$ [Wang A321173]

7) $-1,-4,5,-15,31,-72,160,-361,810,-1821, \cdots \quad$ [Wang A321174]

8) $1,0,0,1,-2,5,-11,25,-56,126, \cdots$ [Sloane A006054]

9) $1,-2,4,-9,20,-45,101,-227,510,-1146, \cdots \quad$ [A052534, Deutsch A109110]

10) $1,0,2,-3,8,-17,39,-87,196,-440, \cdots \quad$ [Pharo A219788]

\section{Integer Sequences-S3}

In this section, let

$$
X=\frac{\sin (2 \theta)}{\sin (8 \theta)}, Y=\frac{\sin (4 \theta)}{\sin (2 \theta)}, Z=\frac{\sin (8 \theta)}{\sin (4 \theta)} .
$$

Proposition 24. With above notations, $\{X, Y, Z\}$ are the roots of the integer equation

$$
x^{3}+x^{2}-2 x-1=0
$$


Proof.

$$
\begin{gathered}
X+Y+Z=\sum \frac{\sin (2 \theta)}{\sin (8 \theta)}=\frac{1}{P} W(2,1)=-1, \\
X Y+Y Z+Z X=\sum\left(\frac{\sin (2 \theta)}{\sin (8 \theta)}\right)\left(\frac{\sin (4 \theta)}{\sin (2 \theta)}\right)=\frac{1}{P} W(1,2)=-2, \\
X Y Z=\left(\frac{\sin (2 \theta)}{\sin (8 \theta)}\right)\left(\frac{\sin (4 \theta)}{\sin (2 \theta)}\right)\left(\frac{\sin (8 \theta)}{\sin (4 \theta)}\right)=1 .
\end{gathered}
$$

It follows that $\{X, Y, Z\}$ are the roots of the Equation (6).

\section{Proposition 25. For case}
1) $u=1, v=1, w=1$.
2) $u=S T S 1, v=S T S 2, w=S T S 3$.
3) $u=S T S 2, v=S T S 3, w=S T S 1$.
4) $u=S T S 3, v=S T S 1, w=S T S 2$.
5) $u=S T C 1, v=S T C 2, w=S T C 3$.
6) $u=S T C 2, v=S T C 3, w=S T C 1$.
7) $u=S T C 3, v=S T C 1, w=S T C 2$.
8) $u=S T T 1, v=S T T 2, w=S T T 3$.
9) $u=S T T 2, v=S T T 3, w=S T T 1$.
10) $u=S T T 3, v=S T T 1, w=S T T 2$.

Then

$$
\left\{p(n)=u X^{n}+v Y^{n}+w Z^{n} \mid n=0,1,2, \cdots\right\}
$$

is an integer sequence with the recurrence relation

$$
p(n)=-p(n-1)+2 p(n-2)+p(n-3) .
$$

Proof. The proof is similar to the proof of Proposition 19.

Example 26. List of associated sequences:

1) $3,-1,5,-4,13,-16,38,-57, \cdots$ [Barry A094648]

2) $0,0,1,-1,3,-4,9,-14,28,-47, \cdots \quad$ [Sloane A006053, Wieder A109509]

3) $0,-1,0,-2,1,-5,5,-14,19,-42, \cdots \quad[\underline{\text { A052547, }}$, Barry A096976 $]$

4) $0,1,-1,3,-4,9,-14,28,-47,89, \cdots \quad[$ Sloane $\underline{\mathrm{A} 006053}$ ]

5) $-1,-2,3,-8,12,-25,41,-79,136,-253, \cdots \quad[$ Wang $\underline{A 321175}]$

6) $-1,-2,-4,-1,-9,3,-22,19,-60,76, \cdots \quad$ [Wang A321461]

7) $-1,5,-4,13,-16,38,-57,117,-193,370, \cdots \quad$ [Barry A094648, A096975]

8) $1,-1,1,-2,3,-6,10,-19,33,-61, \cdots$ [Sloane A028495, Hanna A136752]

9) $1,1,1,2,1,4,0,9,-5,23, \cdots$ [Adamson and Bagula, A122161]

10) $1,-1,3,-4,9,-14,28,-47,89,-155, \cdots \quad$ [Sloane A006053]

\section{Integer Sequences-S4}

In this section, let

$$
X=\frac{\sin ^{2}(2 \theta)}{\sin (4 \theta) \sin (8 \theta)}, Y=\frac{\sin ^{2}(4 \theta)}{\sin (8 \theta) \sin (2 \theta)}, Z=\frac{\sin ^{2}(8 \theta)}{\sin (2 \theta) \sin (4 \theta)} .
$$


Proposition 27. With above notations, $\{X, Y, Z\}$ are the roots of the integer equation

$$
x^{3}+4 x^{2}+3 x-1=0
$$

Proof.

$$
\begin{gathered}
X+Y+Z=\sum \frac{\sin ^{2}(2 \theta)}{\sin (4 \theta) \sin (8 \theta)}=\frac{1}{P} S(3)=-4 \\
X Y+Y Z+Z X=\sum\left(\frac{\sin ^{2}(2 \theta)}{\sin (4 \theta) \sin (8 \theta)}\right)\left(\frac{\sin ^{2}(4 \theta)}{\sin (8 \theta) \sin (2 \theta)}\right) \\
=\frac{1}{P^{2}} W(3,3)=3, \\
X Y Z=\left(\frac{\sin ^{2}(2 \theta)}{\sin (4 \theta) \sin (8 \theta)}\right)\left(\frac{\sin ^{2}(4 \theta)}{\sin (8 \theta) \sin (2 \theta)}\right)\left(\frac{\sin ^{2}(8 \theta)}{\sin (2 \theta) \sin (4 \theta)}\right)=1 .
\end{gathered}
$$

It follows that $\{X, Y, Z\}$ are the roots of the Equation (7).

Proposition 28. For case
1) $u=1, v=1, w=1$.
2) $u=S T S 1, v=S T S 2, w=S T S 3$.
3) $u=S T S 2, v=S T S 3, w=S T S 1$.
4) $u=S T S 3, v=S T S 1, w=S T S 2$.
5) $u=S T C 1, v=S T C 2, w=S T C 3$.
6) $u=S T C 2, v=S T C 3, w=S T C 1$.
7) $u=S T C 3, v=S T C 1, w=S T C 2$.
8) $u=S T T 1, v=S T T 2, w=S T T 3$.
9) $u=S T T 2, v=S T T 3, w=S T T 1$.
10) $u=S T T 3, v=S T T 1, w=S T T 2$.

Then

$$
\left\{p(n)=u X^{n}+v Y^{n}+w Z^{n} \mid n=0,1,2, \cdots\right\}
$$

is an integer sequence with the recurrence relation

$$
p(n)=-4 p(n-1)-3 p(n-2)+p(n-3) .
$$

Proof. The proof is similar to the proof of Proposition 19.

Example 29. List of associated sequences:
1) $3,-4,10,-25,66,-179,493, \cdots$ [Wang A274220]
2) $0,-1,2,-5,13,-35,96,-266,741,-2070, \cdots$ [Delham A085810]
3) $0,1,-3,9,-26,74,-209,588,-1651,4631, \cdots \quad$ [Adamson $\underline{\text { A116423 }}$ ]
4) $0,0,1,-4,13,-39,113,-322,910,-2561, \cdots$ [Witula A215404]
5) $-1,-1,-1,6,-22,69,-204,587,-1667,4703, \cdots$
6) $-1,6,-15,41,-113,314,-876,2449,-6854,19193, \cdots$
7) $-1,-1,6,-22,69,-204,587,-1667,4703,-13224, \cdots$
8) $1,0,0,1,-4,13,-39,113,-322,910, \cdots$ [Witula A215404]
9) $1,-2,6,-17,48,-135,379,-1063,2980,-8352, \cdots \quad$ [Butler A136776]
10) $1,-2,4,-9,22,-57,153,-419,1160,-3230, \cdots \quad$ [Wang A322504] 


\section{Integer Sequences-S5}

In this section, let

$$
X=\frac{\sin (4 \theta) \sin (8 \theta)}{\sin ^{2}(2 \theta)}, Y=\frac{\sin (8 \theta) \sin (2 \theta)}{\sin ^{2}(4 \theta)}, Z=\frac{\sin (2 \theta) \sin (4 \theta)}{\sin ^{2}(8 \theta)} .
$$

Proposition 30. With above notations, $\{X, Y, Z\}$ are the roots of the integer equation

$$
x^{3}-3 x^{2}-4 x-1=0
$$

Proof.

$$
\begin{gathered}
X+Y+Z=\sum \frac{\sin (4 \theta) \sin (8 \theta)}{\sin ^{2}(2 \theta)}=\frac{1}{P^{2}} W(3,3)=3 . \\
X Y+Y Z+Z X=\sum\left(\frac{\sin (4 \theta) \sin (8 \theta)}{\sin ^{2}(2 \theta)}\right)\left(\frac{\sin (8 \theta) \sin (2 \theta)}{\sin ^{2}(4 \theta)}\right) \\
=\frac{1}{P} S(3)=-4 . \\
X Y Z=\left(\frac{\sin (4 \theta) \sin (8 \theta)}{\sin ^{2}(2 \theta)}\right)\left(\frac{\sin (8 \theta) \sin (2 \theta)}{\sin ^{2}(4 \theta)}\right)\left(\frac{\sin (2 \theta) \sin (4 \theta)}{\sin ^{2}(8 \theta)}\right)=1 .
\end{gathered}
$$

It follows that $\{X, Y, Z\}$ are the roots of the Equation (8).

\section{Proposition 31. For case}

1) $u=1, v=1, w=1$.

2) $u=S T S 1, v=S T S 2, w=S T S 3$.

3) $u=S T S 2, v=S T S 3, w=S T S 1$.

4) $u=S T S 3, v=S T S 1, w=S T S 2$.

5) $u=S T C 1, v=S T C 2, w=S T C 3$.

6) $u=S T C 2, v=S T C 3, w=S T C 1$.

7) $u=S T C 3, v=S T C 1, w=S T C 2$.

8) $u=S T T 1, v=S T T 2, w=S T T 3$.

9) $u=S T T 2, v=S T T 3, w=S T T 1$.

10) $u=S T T 3, v=S T T 1, w=S T T 2$.

Then

$$
\left\{p(n)=u X^{n}+v Y^{n}+w Z^{n} \mid n=0,1,2, \cdots\right\}
$$

is an integer sequence with the recurrence relation

$$
p(n)=3 p(n-1)+4 p(n-2)+p(n-3) .
$$

Proof. The proof is similar to the proof of Proposition 19.

Example 32. List of associated sequences:

1) $3,3,17,66,269,1088,4406, \cdots$ [Witula A215076]

2) $0,-2,-7,-29,-117,-474,-1919,-7770, \cdots$ [Adamson and Bagula A120757]

3) $0,1,4,16,65,263,1065,4312,17459,70690, \cdots$ [Lang A181879]

4) $0,1,3,13,52,211,854,3458,14001,56689, \cdots$ [Bagula and Adamson A122600] 
5) $-1,-8,-29,-120,-484,-1961,-7939,-32145,-130152,-526975, \cdots$

6) $-1,6,20,83,335,1357,5494,22245,90068,364678, \cdots$

7) $-1,-1,-8,-29,-120,-484,-1961,-7939,-32145,-130152, \cdots$

8) $1,3,13,52,211,854,3458,14001,56689,229529, \cdots$ [Bagula and Adamson A122600]

9) $1,1,5,20,81,328,1328,5377,21771,88149, \cdots$

10) $1,-1,-1,-6,-23,-94,-380,-1539,-6231,-25229, \cdots$

\section{Integer Sequences-C1}

In this section, let

$$
X=2 \cos (2 \theta), Y=2 \cos (4 \theta), Z=2 \cos (8 \theta) .
$$

Proposition 33. With above notations, $\{X, Y, Z\}$ are the roots of the integer equation

$$
x^{3}+x^{2}-2 x-1=0
$$

Proof. Using Proposition 7,

$$
\begin{gathered}
X+Y+Z=\sum 2 \cos (2 \theta)=-1 . \\
X Y+Y Z+Z X=\sum(2 \cos (2 \theta))(2 \cos (4 \theta))=-2 . \\
X Y Z=(2 \cos (2 \theta))(2 \cos (4 \theta))(2 \cos (8 \theta))=1 .
\end{gathered}
$$

It follows that $\{X, Y, Z\}$ are the roots of the Equation (9).

\section{Proposition 34. For case}

1) $u=1, v=1, w=1$.

2) $u=S T S 1, v=S T S 2, w=S T S 3$.

3) $u=S T S 2, v=S T S 3, w=S T S 1$.

4) $u=S T S 3, v=S T S 1, w=S T S 2$.

5) $u=S T C 1, v=S T C 2, w=S T C 3$.

6) $u=S T C 2, v=S T C 3, w=S T C 1$.

7) $u=S T C 3, v=S T C 1, w=S T C 2$.

8) $u=S T T 1, v=S T T 2, w=S T T 3$.

9) $u=S T T 2, v=S T T 3, w=S T T 1$.

10) $u=S T T 3, v=S T T 1, w=S T T 2$.

Then

$$
\left\{p(n)=u X^{n}+v Y^{n}+w Z^{n} \mid n=0,1,2, \cdots\right\}
$$

is an integer sequence with the recurrence relation

$$
p(n)=-p(n-1)+2 p(n-2)+p(n-3) .
$$

Proof. We will prove case 4, 5, 8. The proof for other cases are similar.

Case 4: By Lemma 14, $p(0)=0$.

$$
p(1)=u X+v Y+w Z=\sum\left(\frac{1}{2 \sqrt{7} \sin (8 \theta)}\right)(2 \cos (2 \theta))
$$




$$
\begin{gathered}
=\frac{1}{\sqrt{7}} \sum\left(\frac{1}{\sin (8 \theta)}\right)\left(\frac{\sin (4 \theta)}{2 \sin (2 \theta)}\right) \\
=\frac{1}{2 \sqrt{7}} \sum \frac{\sin (4 \theta)}{\sin (2 \theta) \sin (8 \theta)} \\
=\frac{1}{2 P \sqrt{7}} \sum \sin ^{2}(4 \theta)=\frac{1}{2 P \sqrt{7}} S(2)=-1 . \\
p(2)=u X^{2}+v Y^{2}+w Z^{2}=\sum\left(\frac{1}{2 \sqrt{7} \sin (8 \theta)}\right)(2 \cos (2 \theta))^{2} \\
=\frac{2}{\sqrt{7}} \sum\left(\frac{1}{\sin (8 \theta)}\right)\left(\frac{\sin (4 \theta)}{2 \sin (2 \theta)}\right)^{2} \\
=\frac{1}{2 \sqrt{7}} \sum \frac{\sin ^{2}(4 \theta)}{\sin ^{2}(2 \theta) \sin (8 \theta)}=\frac{1}{2 P^{2} \sqrt{7}} W(4,1)=0 .
\end{gathered}
$$

Case 5: By Lemma 15, $p(0)=-1$.

$$
\begin{aligned}
p(1) & =u X+v Y+w Z=\sum(2 \cos (2 \theta))(2 \cos (2 \theta)) \\
& =4 \sum \cos ^{2}(2 \theta)=4 C(2)=5 . \\
p(2)= & u X^{2}+v Y^{2}+w Z^{2}=\sum(2 \cos (2 \theta))(2 \cos (2 \theta))^{2} \\
= & 8 \sum \cos ^{3}(2 \theta)=8 C(3)=-4 .
\end{aligned}
$$

Case 8: By Lemma 16, $p(0)=1$.

$$
\begin{aligned}
p(1) & =u X+v Y+w Z=\sum\left(\frac{1}{\sqrt{7} \tan (2 \theta)}\right)(2 \cos (2 \theta)) \\
& =\frac{2}{\sqrt{7}} \sum\left(\frac{1}{\tan (2 \theta)}\right)(\cos (2 \theta)) \\
& =\frac{2}{\sqrt{7}} \sum\left(\frac{\sin (4 \theta)}{2 \sin ^{2}(2 \theta)}\right)\left(\frac{\sin (4 \theta)}{2 \sin (2 \theta)}\right) \\
& =\frac{1}{2 \sqrt{7}} \sum \frac{\sin ^{2}(4 \theta)}{\sin ^{3}(2 \theta)}=\frac{1}{2 P^{3} \sqrt{7}} W(5,3)=-1 \\
p(2) & =u X^{2}+v Y^{2}+w Z^{2}=\sum\left(\frac{1}{\sqrt{7} \tan (2 \theta)}\right)(2 \cos (2 \theta))^{2} \\
& =\frac{4}{\sqrt{7}} \sum\left(\frac{1}{\tan ^{2}(2 \theta)}\right)(\cos (2 \theta))^{2} \\
& =\frac{4}{\sqrt{7}} \sum\left(\frac{\sin ^{2}(4 \theta)}{2 \sin ^{2}(2 \theta)}\right)\left(\frac{\sin (4 \theta)}{2 \sin (2 \theta)}\right)^{2} \\
& =\frac{1}{2 \sqrt{7}} \sum \frac{\sin ^{3}(4 \theta)}{\sin ^{4}(2 \theta)}=\frac{1}{2 P^{4} \sqrt{7}} W(7,4)=3 .
\end{aligned}
$$

Example 35. List of associated sequences:

1) $3,-1,5,-4,13,-16,38,-57, \cdots$ [Barry A094648]

2) $0,1,-1,3,-4,9,-14,28,-47,89, \cdots \quad$ [Sloane A006053] 
3) $0,0,1,-1,3,-4,9,-14,28,-47, \cdots \quad$ [Sloane A $\underline{\text { 0006053 }}$ ]

4) $0,-1,0,-2,1,-5,5,-14,19,-42, \cdots$ [Barry A052547, A096976]

5) $-1,5,-4,13,-16,38,-57,117,-193,370, \cdots \quad$ [Barry $\underline{A 094648}, \underline{A 096975}]$

6) $-1,-2,3,-8,12,-25,41,-79,136,-253, \cdots$

7) $-1,-2,-4,-1,-9,3,-22,19,-60,76, \cdots$

8) $1,-1,3,-4,9,-14,28,-47,89,-155, \cdots \quad$ [Sloane A006053]

9) $1,-1,1,-2,3,-6,10,-19,33,-61, \cdots$ [Sloane A028495, Hanna A136752]

10) $1,1,1,2,1,4,0,9,-5,23, \cdots$ [Adamson and Bagula A122161]

\section{Integer Sequences-C2}

In this section, let

$$
X=\frac{\cos (2 \theta)}{\cos (4 \theta)}, Y=\frac{\cos (4 \theta)}{\cos (8 \theta)}, Z=\frac{\cos (8 \theta)}{\cos (2 \theta)} .
$$

Proposition 36. With above notations, $\{X, Y, Z\}$ are the roots of the integer equation

$$
x^{3}+4 x^{2}+3 x-1=0
$$

Proof.

$$
\begin{aligned}
& X+Y+Z=\sum \frac{\cos (2 \theta)}{\cos (4 \theta)}=\sum\left(\frac{\sin (4 \theta)}{2 \sin (2 \theta)}\right)\left(\frac{2 \sin (4 \theta)}{\sin (8 \theta)}\right) \\
&= \sum \frac{\sin ^{2}(4 \theta)}{\sin (2 \theta) \sin (8 \theta)}=\frac{1}{P} \sum \sin ^{3}(2 \theta) \\
&= \frac{1}{P} S(3)=-4 . \\
& X Y+Y Z+Z X=\sum \frac{\cos (2 \theta)}{\cos (4 \theta)} \frac{\cos (4 \theta)}{\cos (8 \theta)}=\sum \frac{\cos (2 \theta)}{\cos (8 \theta)} \\
&=\sum\left(\frac{\sin (4 \theta)}{2 \sin (2 \theta)}\right)\left(\frac{2 \sin (8 \theta)}{\sin (16 \theta)}\right) \\
&=\sum \frac{\sin (4 \theta) \sin (8 \theta)}{\sin ^{2}(2 \theta)}=\frac{1}{P^{2}} W(3,3)=3 . \\
& X Y Z=\left(\frac{\cos (2 \theta)}{\cos (4 \theta)}\right)\left(\frac{\cos (4 \theta)}{\cos (8 \theta)}\right)\left(\frac{\cos (8 \theta)}{\cos (2 \theta)}\right)=1 .
\end{aligned}
$$

It follows that $\{X, Y, Z\}$ are the roots of the Equation (10).

\section{Proposition 37. For case}
1) $u=1, v=1, w=1$.
2) $u=S T S 1, v=S T S 2, w=S T S 3$.
3) $u=S T S 2, v=S T S 3, w=S T S 1$.
4) $u=S T S 3, v=S T S 1, w=S T S 2$.
5) $u=S T C 1, v=S T C 2, w=S T C 3$.
6) $u=S T C 2, v=S T C 3, w=S T C 1$.
7) $u=S T C 3, v=S T C 1, w=S T C 2$. 
8) $u=S T T 1, v=S T T 2, w=S T T 3$.

9) $u=S T T 2, v=S T T 3, w=S T T 1$.

10) $u=S T T 3, v=S T T 1, w=S T T 2$.

Then

$$
\left\{p(n)=u X^{n}+v Y^{n}+w Z^{n} \mid n=0,1,2, \cdots\right\}
$$

is an integer sequence with the recurrence relation

$$
p(n)=-4 p(n-1)=3 p(n-2)+p(n-3) .
$$

Proof. The proof is similar to the proof of Proposition 34.

Example 38. List of associated sequence:

1) $3,-4,10,-25,66,-179,493, \cdots$ [Wang $\underline{\text { A274220] }}$

2) $0,0,1,-4,13,-39,113,-322,910,-2561, \cdots$ [Witula A215404]

3) $0,-1,2,-5,13,-35,96,-266,741,-2070, \cdots \quad$ [Delham $\underline{\text { A085810] }}$

4) $0,1,-3,9,-26,74,-209,588,-1651,4631, \cdots$ [Adamson $\underline{\text { A116423] }}$

5) $-1,-1,6,-22,69,-204,587,-1667,4703,-13224, \cdots$

6) $-1,-1,-1,6,-22,69,-204,587,-1667,4703, \cdots$

7) $-1,6,-15,41,-113,314,-876,2449,-6854,19193, \cdots$

8) $1,-2,4,-9,22,-57,153,-419,1160,-3230, \cdots$

9) $1,0,0,1,-4,13,-39,113,-322,910, \cdots$ [Witula A215404]

10) $1,-2,6,-17,48,-135,379,-1063,2980,-8352, \cdots \quad$ [Butler A136776]

\section{Integer Sequences-C3}

In this section, let

$$
X=\frac{\cos (2 \theta)}{\cos (8 \theta)}, Y=\frac{\cos (4 \theta)}{\cos (2 \theta)}, Z=\frac{\cos (8 \theta)}{\cos (4 \theta)} .
$$

Proposition 39. With above notations, $\{X, Y, Z\}$ are the roots of the integer equation

$$
x^{3}-3 x^{2}-4 x-1=0
$$

Proof.

$$
\begin{aligned}
X+Y+Z & =\sum \frac{\cos (2 \theta)}{\cos (8 \theta)}=\sum\left(\frac{\sin (4 \theta)}{2 \sin (2 \theta)}\right)\left(\frac{2 \sin (8 \theta)}{\sin (16 \theta)}\right) \\
= & \sum \frac{\sin (4 \theta) \sin (8 \theta)}{\sin ^{2}(2 \theta)}=\frac{1}{P^{2}} W(3,3)=3 \\
X Y+Y Z+Z X & =\sum\left(\frac{\cos (2 \theta)}{\cos (8 \theta)}\right)\left(\frac{\cos (4 \theta)}{\cos (2 \theta)}\right)=\sum \frac{\cos (4 \theta)}{\cos (8 \theta)} \\
& =\sum\left(\frac{\sin (8 \theta)}{2 \sin (4 \theta)}\right)\left(\frac{2 \sin (8 \theta)}{\sin (16 \theta)}\right) \\
& =\sum \frac{\sin ^{2}(8 \theta)}{\sin (4 \theta) \sin (2 \theta)}=\frac{1}{P} S(3)=-4
\end{aligned}
$$




$$
X Y Z=\left(\frac{\cos (2 \theta)}{\cos (8 \theta)}\right)\left(\frac{\cos (4 \theta)}{\cos (2 \theta)}\right)\left(\frac{\cos (8 \theta)}{\cos (4 \theta)}\right)=1
$$

It follows that $\{X, Y, Z\}$ are the roots of the Equation (11).

Proposition 40. For case
1) $u=1, v=1, w=1$.
2) $u=S T S 1, v=S T S 2, w=S T S 3$.
3) $u=S T S 2, v=S T S 3, w=S T S 1$.
4) $u=S T S 3, v=S T S 1, w=S T S 2$.
5) $u=S T C 1, v=S T C 2, w=S T C 3$
6) $u=S T C 2, v=S T C 3, w=S T C 1$.
7) $u=S T C 3, v=S T C 1, w=S T C 2$.
8) $u=S T T 1, v=S T T 2, w=S T T 3$.
9) $u=S T T 2, v=S T T 3, w=S T T 1$.
10) $u=S T T 3, v=S T T 1, w=S T T 2$.

Then

$$
\left\{p(n)=u X^{n}+v Y^{n}+w Z^{n} \mid n=0,1,2, \cdots\right\}
$$

is an integer sequence with the recurrence relation

$$
p(n)=3 p(n-1)+4 p(n-2)+p(n-3) .
$$

Proof. The proof is similar to the proof of Proposition 34.

Example 41. List of associated sequences:

1) $3,3,17,66,269,1088,4406,17839, \cdots$ [Witula A215076]

2) $0,-2,-7,-29,-117,-474,-1919,-7770, \cdots$ [Adamson and Bagula A120757]

3) $0,1,4,16,65,263,1065,4312,17459,70690, \cdots$ [Lang A181879]

4) $0,1,3,13,52,211,854,3458,14001,56689, \cdots$ [Bagula and Adamson $\underline{\text { A122600] }}$

5) $-1,-8,-29,-120,-484,-1961,-7939,-32145,-130152,-526975, \cdots$

6) $-1,6,20,83,335,1357,5494,22245,90068,364678, \cdots$

7) $-1,-1,-8,-29,-120,-484,-1961,-7939,-32145,-130152, \cdots$

8) $1,3,13,52,211,854,3458,14001,56689,229529, \cdots$ [Bagula and Adamson A122600]

9) $1,1,5,20,81,328,1328,5377,21771,88149, \cdots$

10) $1,-1,-1,-6,-23,-94,-380,-1539,-6231,-25229, \cdots$

\section{Integer Sequences-C4}

In this section, let

$$
X=\frac{\cos ^{2}(2 \theta)}{\cos (4 \theta) \cos (8 \theta)}, Y=\frac{\cos ^{2}(4 \theta)}{\cos (8 \theta) \cos (2 \theta)}, Z=\frac{\cos ^{2}(8 \theta)}{\cos (2 \theta) \cos (4 \theta)} .
$$

Proposition 42. With above notations, $\{X, Y, Z\}$ are the roots of the integer equation

$$
x^{3}+4 x^{2}-11 x-1=0
$$


Proof.

$$
\begin{aligned}
& X+Y+Z= \sum \frac{\cos ^{2}(2 \theta)}{\cos (4 \theta) \cos (8 \theta)} \\
&= \sum\left(\frac{\sin (4 \theta)}{2 \sin (2 \theta)}\right)^{2}\left(\frac{2 \sin (4 \theta)}{\sin (8 \theta)}\right)\left(\frac{2 \sin (8 \theta)}{\sin (16 \theta)}\right) \\
&= \sum \frac{\sin ^{3}(4 \theta)}{\sin ^{3}(2 \theta)}=\frac{1}{P 3} W(6,3)=-4 \\
& X Y+Y Z+Z X=\sum\left(\frac{\cos ^{2}(2 \theta)}{\cos (4 \theta) \cos (8 \theta)}\right)\left(\frac{\cos ^{2}(4 \theta)}{\cos (8 \theta) \cos (2 \theta)}\right) \\
&=\sum \frac{\cos (2 \theta) \cos (4 \theta)}{\cos ^{2}(8 \theta)} \\
&=\sum\left(\frac{\sin ^{2}(4 \theta)}{2 \sin ^{2}(2 \theta)}\right)\left(\frac{\sin (8 \theta)}{2 \sin (4 \theta)}\right)\left(\frac{2 \sin (8 \theta)}{\sin (16 \theta)}\right)^{2} \\
&=\sum \frac{\sin ^{3}(8 \theta)}{\sin ^{3}(2 \theta)}=\frac{1}{P 3} W(3,6)=-11 \\
& X Y Z=\sum\left(\frac{\cos ^{2}(2 \theta)}{\cos (4 \theta) \cos (8 \theta)}\right)\left(\frac{\cos ^{2}(4 \theta)}{\cos (8 \theta) \cos (2 \theta)}\right)\left(\frac{\cos ^{2}(8 \theta)}{\cos (2 \theta) \cos (4 \theta)}\right)=1
\end{aligned}
$$

It follows that $\{X, Y, Z\}$ are the roots of the Equation (12).

Proposition 43. For case
1) $u=1, v=1, w=1$.
2) $u=S T S 1, v=S T S 2, w=S T S 3$.
3) $u=S T S 2, v=S T S 3, w=S T S 1$.
4) $u=S T S 3, v=S T S 1, w=S T S 2$.
5) $u=S T C 1, v=S T C 2, w=S T C 3$.
6) $u=S T C 2, v=S T C 3, w=S T C 1$.
7) $u=S T C 3, v=S T C 1, w=S T C 2$.
8) $u=S T T 1, v=S T T 2, w=S T T 3$.
9) $u=S T T 2, v=S T T 3, w=S T T 1$.
10) $u=S T T 3, v=S T T 1, w=S T T 2$.

Then

$$
\left\{p(n)=u X^{n}+v Y^{n}+w Z^{n} \mid n=0,1,2, \cdots\right\}
$$

is an integer sequence with the recurrence relation

$$
p(n)=-4 p(n-1)+11 p(n-2)+p(n-3) .
$$

Proof. The proof is similar to the proof of Proposition 34.

Example 44. List of associated sequences:
1) $3,-4,38,-193,1186,-6829,40169, \cdots$ [Wang A274663]
2) $0,3,-14,89,-507,2993,-17460,102256,-598091,3499720, \cdots$
3) $0,-1,9,-47,286,-1652,9707,-56714,331981,-1942071, \cdots$
4) $0,-2,5,-42,221,-1341,7753,-45542,266110,-1557649, \cdots$ 
5) $-1,13,-57,370,-2094,12389,-72220,423065,-2474291,14478659, \cdots$

6) $-1,-8,41,-253,1455,-8562,50000,-292727,1712346,-10019381, \cdots$

7) $-1,-1,-22,76,-547,3002,-17949,104271,-611521,3575116, \cdots$

8) $1,-4,28,-155,924,-5373,31501,-184183,1077870,-6305992, \cdots$

9) $1,-2,10,-61,352,-2069,12087,-70755,413908,-2421850, \cdots$

10) $1,2,0,23,-90,613,-3419,20329,-118312,693448, \cdots$

\section{Integer Sequences-C5}

In this section, let

$$
X=\frac{\cos (4 \theta) \cos (8 \theta)}{\cos ^{2}(2 \theta)}, Y=\frac{\cos (8 \theta) \cos (2 \theta)}{\cos ^{2}(4 \theta)}, Z=\frac{\cos (2 \theta) \cos (4 \theta)}{\cos ^{2}(8 \theta)} .
$$

Proposition 45. With above notations, $\{X, Y, Z\}$ are the roots of the integer equation

$$
x^{3}+11 x^{2}-4 x-1=0
$$

Proof.

$$
\begin{aligned}
& X+Y+Z=\sum \frac{\cos (4 \theta) \cos (8 \theta)}{\cos ^{2}(2 \theta)} \\
&=\sum\left(\frac{\sin (8 \theta)}{2 \sin (4 \theta)}\right)\left(\frac{\sin (16 \theta)}{2 \sin (8 \theta)}\right)\left(\frac{2 \sin (2 \theta)}{\sin (4 \theta)}\right)^{2} \\
&=\sum \frac{\sin ^{3}(2 \theta)}{\sin ^{3}(4 \theta)}=\frac{1}{P 3} W(3,6)=-11 \\
& X Y+Y Z+Z X=\sum\left(\frac{\cos (4 \theta) \cos (8 \theta)}{\cos ^{2}(2 \theta)}\right)\left(\frac{\cos (8 \theta) \cos (2 \theta)}{\cos ^{2}(4 \theta)}\right) \\
&=\sum \frac{\cos ^{2}(8 \theta)}{\cos (2 \theta) \cos (4 \theta)} \\
&=\sum\left(\frac{\sin ^{2}(16 \theta)}{2 \sin ^{2}(8 \theta)}\right)^{2}\left(\frac{2 \sin (2 \theta)}{\sin (4 \theta)}\right)\left(\frac{2 \sin (4 \theta)}{\sin ^{2}(8 \theta)}\right) \\
&=\sum \frac{\sin ^{3}(2 \theta)}{\sin ^{3}(8 \theta)}=\frac{1}{P 3} W(6,3)=-4 \\
& X Y Z=\sum\left(\frac{\cos (4 \theta) \cos (8 \theta)}{\cos ^{2}(2 \theta)}\right)\left(\frac{\cos (8 \theta) \cos (2 \theta)}{\cos ^{2}(4 \theta)}\right)\left(\frac{\cos (2 \theta) \cos (4 \theta)}{\cos ^{2}(8 \theta)}\right)=1
\end{aligned}
$$

It follows that $\{X, Y, Z\}$ are the roots of the Equation (13).

Proposition 46. For case
1) $u=1, v=1, w=1$.
2) $u=S T S 1, v=S T S 2, w=S T S 3$.
3) $u=S T S 2, v=S T S 3, w=S T S 1$.
4) $u=S T S 3, v=S T S 1, w=S T S 2$.
5) $u=S T C 1, v=S T C 2, w=S T C 3$.
6) $u=S T C 2, v=S T C 3, w=S T C 1$. 
7) $u=S T C 3, v=S T C 1, w=S T C 2$.

8) $u=S T T 1, v=S T T 2, w=S T T 3$.

9) $u=S T T 2, v=S T T 3, w=S T T 1$.

10) $u=S T T 3, v=S T T 1, w=S T T 2$.

Then

$$
\left\{p(n)=u X^{n}+v Y^{n}+w Z^{n} \mid n=0,1,2, \cdots\right\}
$$

is an integer sequence with the recurrence relation

$$
p(n)=-11 p(n-1)+4 p(n-2)+p(n-3) .
$$

Proof. The proof is similar to the proof of Proposition 34.

Example 47. List of associated sequences:
1) $3,-11,129,-1460,165655,-187926,2131986, \cdots$ [Wang A274663]
2) $0,-2,25,-283,3211,-36428,413269,-4688460,53189708,-603427359, \cdots$
3) $0,5,-56,636,-7215,81853,-928607,10534874,-119516189$,
3) $1355888968, \cdots$
4) $0,-3,31,-353,4004,-45425,515338,-5846414,66326481,-752461609, \cdots$
5) $-1,6,-57,650,-7372,83635,-948823,10764221,-122118088$,
$1385407029, \cdots$
6) $-1,20,-232,2631,-29849,338631,-3841706,43583441,-494446044$, $5609398542, \cdots$
7) $-1,-15,160,-1821,20656,-234340,2658543,-30160677,342167279, \cdots$
8) $1,1,-11,126,-1429,16212,-183922,2086561,-23671647,268550439, \cdots$
9) $1,-9,101,-1146,13001,-147494,1673292,-18983187,215360731$, $-2443227497, \cdots$
10) $1,-3,39,-440,4993,-56644,642616,-7290359,82707769,-938304279, \cdots$

\section{Integer Sequences-T1}

In this section, let

$$
X=\tan ^{2}(2 \theta), Y=\tan ^{2}(4 \theta), Z=\tan ^{2}(8 \theta) .
$$

Proposition 48. With above notations, $\{X, Y, Z\}$ are the roots of the integer equation

$$
x^{3}-21 x^{2}+35 x-7=0 \text {. }
$$

Proof.

$$
\begin{aligned}
& X+Y+Z=\sum \tan ^{2}(2 \theta)=T(2)=21 . \\
& X Y+Y Z+Z X=\sum \tan ^{2}(2 \theta) \tan ^{2}(4 \theta) \\
&=(\tan (2 \theta) \tan (4 \theta) \tan (8 \theta))^{2} \sum \frac{1}{\tan ^{2}(2 \theta)} \\
&=(-\sqrt{7})^{2} \sum\left(\frac{\sin (4 \theta)}{2 \sin ^{2}(2 \theta)}\right)^{2} \\
&=\frac{7}{4} \sum \frac{\sin ^{2}(4 \theta)}{\sin ^{4}(2 \theta)}=\frac{7}{4 P 4} W(6,4)=35 .
\end{aligned}
$$




$$
X Y Z=(\tan (2 \theta) \tan (4 \theta) \tan (8 \theta))^{2}=7 .
$$

It follows that $\{X, Y, Z\}$ are the roots of the Equation (14).

Proposition 49. For case

1) $u=1, v=1, w=1$.

2) $u=S T S 1, v=S T S 2, w=S T S 3$.

3) $u=S T S 2, v=S T S 3, w=S T S 1$.

4) $u=S T S 3, v=S T S 1, w=S T S 2$.

5) $u=S T C 1, v=S T C 2, w=S T C 3$.

6) $u=S T C 2, v=S T C 3, w=S T C 1$.

7) $u=S T C 3, v=S T C 1, w=S T C 2$.

8) $u=S T T 1, v=S T T 2, w=S T T 3$.

9) $u=S T T 2, v=S T T 3, w=S T T 1$.

10) $u=S T T 3, v=S T T 1, w=S T T 2$.

Then

$$
\left\{p(n)=u X^{n}+v Y^{n}+w Z^{n} \mid n=0,1,2, \cdots\right\}
$$

is an integer sequence with the recurrence relation

$$
p(n)=21 p(n-1)-35 p(n-2)+7 p(n-3) .
$$

Proof. We will prove case 4, 7, 10 and the other cases are similar.

Case 4: By Lemma 14, $p(0)=0$.

$$
\begin{aligned}
p(1) & =u X+v Y+w Z=\sum\left(\frac{1}{2 \sqrt{7} \sin (8 \theta)}\right)\left(\tan ^{2}(2 \theta)\right) \\
& =\frac{1}{2 \sqrt{7}} \sum\left(\frac{1}{\sin (8 \theta)}\right)\left(\frac{2 \sin ^{2}(2 \theta)}{\sin (4 \theta)}\right)^{2} \\
& =\frac{2}{\sqrt{7}} \sum \frac{\sin ^{4}(2 \theta)}{\sin ^{2}(4 \theta) \sin (8 \theta)}=\frac{2}{P^{2} \sqrt{7}} W(1,6)=4 . \\
p(2)= & u X^{2}+v Y^{2}+w Z^{2}=\sum\left(\frac{1}{2 \sqrt{7} \sin (8 \theta)}\right)\left(\tan ^{2}(2 \theta)\right)^{2} \\
= & \frac{1}{2 \sqrt{7}} \sum\left(\frac{1}{\sin ^{2}(8 \theta)}\right)\left(\frac{2 \sin ^{2}(2 \theta)}{\sin ^{4}(4 \theta)}\right)^{4} \\
= & \frac{8}{\sqrt{7}} \sum \frac{\sin ^{8}(2 \theta)}{\sin ^{4}(4 \theta) \sin (8 \theta)}=\frac{2}{P^{4} \sqrt{7}} W(3,12)=88 .
\end{aligned}
$$

Case 7: By Lemma 15, $p(0)=-1$.

$$
\begin{aligned}
p(1) & =u X+v Y+w Z=\sum(2 \cos (8 \theta))\left(\tan ^{2}(2 \theta)\right) \\
& =2 \sum\left(\frac{\sin (16 \theta)}{2 \sin (8 \theta)}\right)\left(\frac{2 \sin ^{2}(2 \theta)}{\sin (4 \theta)}\right)^{2} \\
& =4 \sum \frac{\sin ^{5}(2 \theta)}{\sin (8 \theta) \sin ^{2}(4 \theta)}=\frac{4}{P^{2}} W(1,7)=21
\end{aligned}
$$




$$
\begin{aligned}
p(2) & =u X^{2}+v Y^{2}+w Z^{2}=\sum(2 \cos (8 \theta))\left(\tan ^{2}(2 \theta)\right)^{2} \\
& =2 \sum\left(\frac{\sin (16 \theta)}{2 \sin (8 \theta)}\right)\left(\frac{2 \sin ^{2}(2 \theta)}{\sin (4 \theta)}\right)^{4} \\
& =16 \sum \frac{\sin ^{9}(2 \theta)}{\sin (8 \theta) \sin ^{4}(4 \theta)}=\frac{16}{P^{4}} W(3,13)=455 .
\end{aligned}
$$

Case 10: By Lemma 16, $p(0)=1$.

$$
\begin{aligned}
p(1) & =u X+v Y+w Z=\sum\left(\frac{1}{\sqrt{7} \tan (8 \theta)}\right)\left(\tan ^{2}(2 \theta)\right) \\
& =\frac{1}{\sqrt{7}} \sum\left(\frac{\sin (16 \theta)}{2 \sin ^{2}(8 \theta)}\right)\left(\frac{2 \sin ^{2}(2 \theta)}{\sin (4 \theta)}\right)^{2} \\
& =\frac{2}{\sqrt{7}} \sum \frac{\sin ^{5}(2 \theta)}{\sin ^{2}(4 \theta) \sin ^{2}(8 \theta)}=\frac{2}{P^{2} \sqrt{7}} S(7)=7 . \\
p(2) & =u X^{2}+v Y^{2}+w Z^{2}=\sum\left(\frac{1}{\sqrt{7} \tan (8 \theta)}\right)\left(\tan ^{2}(2 \theta)\right)^{2} \\
& =\frac{1}{\sqrt{7}} \sum\left(\frac{\sin ^{2}(16 \theta)}{2 \sin ^{2}(8 \theta)}\right)\left(\frac{2 \sin { }^{2}(2 \theta)}{\sin (4 \theta)}\right)^{4} \\
& =\frac{2}{\sqrt{7}} \sum \frac{\sin ^{9}(2 \theta)}{\sin ^{4}(4 \theta) \sin ^{2}(8 \theta)}=\frac{8}{P^{4} \sqrt{7}} W(2,13)=113 .
\end{aligned}
$$

Example 50. List of associated sequences.

1) $3,21,371,7077,135779,2606261, \cdots$ [Delham A108716]

2) $0,4,72,1372,26320,505204,9697688,186153548,3573341856$, $68592688612, \cdots$

3) $0,-8,-160,-3080,-59136,-1135176,-21790496,-418283208, \cdots$

4) $0,4,88,1708,32816,629972,12092808,232129660,4455884384$,

4) $85533683620, \cdots$

5) $-1,-7,-161,-3143,-60417,-1159879,-22264865,-427389319$, $-8204024577, \cdots$

6) $-1,-35,-665,-12747,-244657,-4696307,-90148681, \cdots$

7) $-1,21,455,8813,169295,3249925,62384791,1197518301$, $22987166111, \cdots$

8) $1,-1,-31,-609,-11711,-224833,-4315871,-82846113, \cdots$ [Witula A215794]

9) $1,15,289,5551,106561,2045519,39265121,753720303,14468165761$, $277726126223, \cdots$

10) $1,7,113,2135,40929,785575,15079505,289460983,5556396993$, $106658758983, \cdots$

\section{Integer Sequences-T2}

In this section, let 


$$
X=\frac{\tan (2 \theta)}{\tan (4 \theta)}, Y=\frac{\tan (4 \theta)}{\tan (8 \theta)}, Z=\frac{\tan (8 \theta)}{\tan (2 \theta)} .
$$

Proposition 51. With above notations, $\{X, Y, Z\}$ are the roots of the integer equation

$$
x^{3}+9 x^{2}-x-1=0
$$

Proof.

$$
\begin{aligned}
X+Y+Z=\sum \frac{\tan (2 \theta)}{\tan (4 \theta)}=\sum\left(\frac{2 \sin ^{2}(2 \theta)}{\sin (4 \theta)}\right)\left(\frac{\sin (8 \theta)}{2 \sin ^{2}(4 \theta)}\right) \\
=\sum \frac{\sin ^{2}(2 \theta) \sin (8 \theta)}{\sin ^{3}(4 \theta)}=\frac{1}{P^{3}} W(4,5)=-9 . \\
X Y+Y Z+Z X=\sum\left(\frac{\tan (2 \theta)}{\tan (4 \theta)}\right)\left(\frac{\tan (4 \theta)}{\tan (8 \theta)}\right)=\sum \frac{\tan (2 \theta)}{\tan (8 \theta)} \\
=\sum\left(\frac{2 \sin ^{2}(2 \theta)}{\sin ^{2}(4 \theta)}\right)\left(\frac{\sin (16 \theta)}{2 \sin ^{2}(8 \theta)}\right) \\
=\sum \frac{\sin ^{3}(2 \theta)}{\sin (4 \theta) \sin ^{2}(8 \theta)}=\frac{1}{P^{2}} W(5,1)=-1 . \\
X Y Z=\left(\frac{\tan (2 \theta)}{\tan (4 \theta)}\right)\left(\frac{\tan (4 \theta)}{\tan (8 \theta)}\right)\left(\frac{\tan (8 \theta)}{\tan (2 \theta)}\right)=1 .
\end{aligned}
$$

It follows that $\{X, Y, Z\}$ are the roots of the Equation (15).

Proposition 52. For case

1) $u=1, v=1, w=1$.

2) $u=S T S 1, v=S T S 2, w=S T S 3$.

3) $u=S T S 2, v=S T S 3, w=S T S 1$.

4) $u=S T S 3, v=S T S 1, w=S T S 2$.

5) $u=S T C 1, v=S T C 2, w=S T C 3$.

6) $u=S T C 2, v=S T C 3, w=S T C 1$.

7) $u=S T C 3, v=S T C 1, w=S T C 2$.

8) $u=S T T 1, v=S T T 2, w=S T T 3$.

9) $u=S T T 2, v=S T T 3, w=S T T 1$.

10) $u=S T T 3, v=S T T 1, w=S T T 2$.

Then

$$
\left\{p(n)=u X^{n}+v Y^{n}+w Z^{n} \mid n=0,1,2, \cdots\right\}
$$

is an integer sequence with the recurrence relation

$$
p(n)=-9 p(n-1)+p(n-2)+p(n-3) .
$$

Proof. The proof is similar to the proof of Proposition 49.

Example 53. List of associated sequences:

1) $3,-9,83,-753,6851,-62329,567059, \cdots \quad$ [Wang A274032]

2) $0,-2,16,-146,1328,-12082,109920,-1000034,9098144,-82773410, \cdots$

3) $0,4,-36,328,-2984,27148,-246988,2247056,-20443344,185990164, \cdots$ 
4) $0,-2,20,-182,1656,-15066,137068,-1247022,11345200,-103216754, \cdots$

5) $-1,3,-37,335,-3049,27739,-252365,2295975,-20888401,190039219, \cdots$

6) $-1,17,-149,1357,-12345,112313,-1021805,9296213,-84575409$,

$769453089, \cdots$

7) $-1,-11,103,-939,8543,-77723,707111,-6433179,58527999$, $-532478059, \cdots$

8) $1,1,-7,65,-591,5377,-48919,445057,-4049055,36837633, \cdots$

9) $1,-7,65,-591,5377,-48919,445057,-4049055,36837633,-335142695, \cdots$

10) $1,-3,25,-227,2065,-18787,170921,-1555011,14147233,-128709187, \cdots$

\section{Integer Sequences-T3}

In this section, let

$$
X=\frac{\tan (2 \theta)}{\tan (8 \theta)}, Y=\frac{\tan (4 \theta)}{\tan (2 \theta)}, Z=\frac{\tan (8 \theta)}{\tan (4 \theta)} .
$$

Proposition 54. With above notations, $\{X, Y, Z\}$ are the roots of the integer equation

$$
x^{3}+x^{2}-9 x-1=0
$$

Proof.

$$
\begin{aligned}
X+Y+Z & =\sum \frac{\tan (2 \theta)}{\tan (8 \theta)}=\sum\left(\frac{2 \sin ^{2}(2 \theta)}{\sin (4 \theta)}\right)\left(\frac{\sin (16 \theta)}{2 \sin ^{2}(8 \theta)}\right) \\
& =\sum \frac{\sin ^{3}(2 \theta)}{\sin (4 \theta) \sin ^{2}(8 \theta)}=\frac{1}{P^{2}} W(5,1)=-1 . \\
X Y+Y Z+Z X & =\sum\left(\frac{\tan (2 \theta)}{\tan (8 \theta)}\right)\left(\frac{\tan (4 \theta)}{\tan (2 \theta)}\right)=\sum \frac{\tan (4 \theta)}{\tan (8 \theta)} \\
& =\sum \frac{\tan (2 \theta)}{\tan (4 \theta)}=\sum\left(\frac{2 \sin ^{2}(2 \theta)}{\sin ^{2}(4 \theta)}\right)\left(\frac{\sin (8 \theta)}{2 \sin ^{2}(4 \theta)}\right) \\
& =\sum \frac{\sin (2 \theta) \sin (8 \theta)}{\sin ^{3}(4 \theta)}=\frac{1}{P^{3}} W(4,5)=-9 . \\
X Y Z & =\left(\frac{\tan (2 \theta)}{\tan (8 \theta)}\right)\left(\frac{\tan (4 \theta)}{\tan (2 \theta)}\right)\left(\frac{\tan (8 \theta)}{\tan (4 \theta)}\right)=1 .
\end{aligned}
$$

It follows that $\{X, Y, Z\}$ are the roots of the Equation (16).

Proposition 55. For case
1) $u=1, v=1, w=1$.
2) $u=S T S 1, v=S T S 2, w=S T S 3$.
3) $u=S T S 2, v=S T S 3, w=S T S 1$.
4) $u=S T S 3, v=S T S 1, w=S T S 2$.
5) $u=S T C 1, v=S T C 2, w=S T C 3$.
6) $u=S T C 2, v=S T C 3, w=S T C 1$.
7) $u=S T C 3, v=S T C 1, w=S T C 2$.
8) $u=S T T 1, v=S T T 2, w=S T T 3$. 
9) $u=S T T 2, v=S T T 3, w=S T T 1$.

10) $u=S T T 3, v=S T T 1, w=S T T 2$.

Then

$$
\left\{p(n)=u X^{n}+v Y^{n}+w Z^{n} \mid n=0,1,2, \cdots\right\}
$$

is an integer sequence with the recurrence relation

$$
p(n)=-p(n-1)+9 p(n-2)+p(n-3) .
$$

Proof. The proof is similar to the proof of Proposition 49.

\section{Example 56. List of associated sequences:}
1) $3,-1,19,-25,195,-401,2131,-5545, \cdots$ [Wang A274075]
2) $0,0,4,-4,40,-72,428,-1036,4816,-13712, \cdots$ [Librandi A271945]
3) $0,2,-4,22,-56,250,-732,2926,-9264,34866, \cdots$ [Librandi A271944]
4) $0,-2,0,-18,16,-178,304,-1890,4448,-21154, \cdots$
5) $-1,5,3,41,-9,381,-421,3841,-7249,41397, \cdots$
6) $-1,5,-25,69,-289,885,-3417,11093,-40961,137381, \cdots$
7) $-1,-9,3,-85,103,-865,1707,-9389,23887,-106681, \cdots$
8) $1,1,1,9,1,81,-63,793,-1279,8353, \cdots$
9) $1,-3,9,-35,113,-419,1401,-5059,17249,-61379, \cdots$
10) $1,1,9,1,81,-63,793,-1279,8353,-19071, \cdots$

\section{Integer Sequences-T4}

In this section, let

$$
X=\frac{\tan ^{2}(2 \theta)}{\tan (4 \theta) \tan (8 \theta)}, Y=\frac{\tan ^{2}(4 \theta)}{\tan (8 \theta) \tan (2 \theta)}, Z=\frac{\tan ^{2}(8 \theta)}{\tan (2 \theta) \tan (4 \theta)} .
$$

Proposition 57. With above notations, $\{X, Y, Z\}$ are the roots of the integer equation

$$
x^{3}-31 x^{2}-25 x-1=0
$$

Proof.

$$
\begin{aligned}
X+Y+Z & =\sum \frac{\tan ^{2}(2 \theta)}{\tan (4 \theta) \tan (8 \theta)} \\
& =\frac{1}{\tan (2 \theta) \tan (4 \theta) \tan (8 \theta)} \sum \tan ^{3}(2 \theta) \\
& =-\frac{1}{\sqrt{7}} T(3)=31 \\
X Y+Y Z+Z X & =\sum\left(\frac{\tan ^{2}(2 \theta)}{\tan (4 \theta) \tan (8 \theta)}\right)\left(\frac{\tan ^{2}(4 \theta)}{\tan (8 \theta) \tan (2 \theta)}\right) \\
& =\sum \frac{\tan (2 \theta) \tan (4 \theta)}{\tan ^{2}(8 \theta)} \\
& =(\tan (2 \theta) \tan (4 \theta) \tan (8 \theta)) \sum \frac{1}{\tan ^{3}(2 \theta)} \\
& =-\sqrt{7} T(-3)=-25
\end{aligned}
$$




$$
X Y Z=\left(\frac{\tan ^{2}(2 \theta)}{\tan (4 \theta) \tan (8 \theta)}\right)\left(\frac{\tan ^{2}(4 \theta)}{\tan (8 \theta) \tan (2 \theta)}\right)\left(\frac{\tan ^{2}(8 \theta)}{\tan (2 \theta) \tan (4 \theta)}\right)=1 .
$$

It follows that $\{X, Y, Z\}$ are the roots of the Equation (17).

\section{Proposition 58. For case}

1) $u=1, v=1, w=1$.

2) $u=S T S 1, v=S T S 2, w=S T S 3$.

3) $u=S T S 2, v=S T S 3, w=S T S 1$.

4) $u=S T S 3, v=S T S 1, w=S T S 2$.

5) $u=S T C 1, v=S T C 2, w=S T C 3$.

6) $u=S T C 2, v=S T C 3, w=S T C 1$.

7) $u=S T C 3, v=S T C 1, w=S T C 2$.

8) $u=S T T 1, v=S T T 2, w=S T T 3$.

9) $u=S T T 2, v=S T T 3, w=S T T 1$.

10) $u=S T T 3, v=S T T 1, w=S T T 2$.

Then

$$
\left\{p(n)=u X^{n}+v Y^{n}+w Z^{n} \mid n=0,1,2, \cdots\right\}
$$

is an integer sequence with the recurrence relation

$$
p(n)=31 p(n-1)+25 p(n-2)+p(n-3) .
$$

Proof. The proof is similar to the proof of Proposition 49.

Example 59. List of associated sequence:
1) $3,31,1011,32119,1020995,32454831, \cdots$ [Wang A274592]
2) $0,6,196,6226,197912,6291118,199978684,6356815066,202067025264, \cdots$
3) $0,-14,-440,-13990,-444704,-14136014,-449348024,-14283633798, \cdots$
4) $25,8,244,7764,24679$
5)
$-1,-15,-449,-14295,-454385,-14443759,-459130449$, $-14594592279, \cdots$
6) $-1,-57,-1821,-57877,-1839769,-58481585,-1858981237$, $-59092297741, \cdots$
7) $-1,41,1259,40053,1273159,40470513,1286454931,40893138845$, $1299889147983, \cdots$
8) $1,-3,-87,-2771,-88079,-2799811,-88998887,-2829048851$, $-89928286367, \cdots$
9) $1,25,793,25209,801329,25472217,809697161,25738218745$, $818152682337, \cdots$ 10) $1,9,305,9681,307745,9782425,310958481,9884581281$, $314205764161, \cdots$

\section{Integer Sequences-T5}

In this section, let

$$
X=\frac{\tan (4 \theta) \tan (8 \theta)}{\tan ^{2}(2 \theta)}, Y=\frac{\tan (8 \theta) \tan (2 \theta)}{\tan ^{2}(4 \theta)}, Z=\frac{\tan (2 \theta) \tan (4 \theta)}{\tan ^{2}(8 \theta)} .
$$


Proposition 60. With above notations, $\{X, Y, Z\}$ are the roots of the integer equation

$$
x^{3}+25 x^{2}+31 x-1=0
$$

Proof.

$$
\begin{aligned}
& X+Y+Z=\sum \frac{\tan (4 \theta) \tan (8 \theta)}{\tan ^{2}(2 \theta)} \\
&=(\tan (2 \theta) \tan (4 \theta) \tan (8 \theta)) \sum \frac{1}{\tan ^{3}(2 \theta)} \\
&=-\sqrt{7} T(-3)=-25 \\
& X Y+Y Z+Z X=\sum\left(\frac{\tan (4 \theta) \tan (8 \theta)}{\tan ^{2}(2 \theta)}\right)\left(\frac{\tan (8 \theta) \tan (2 \theta)}{\tan ^{2}(4 \theta)}\right) \\
&=\sum \frac{\tan ^{2}(8 \theta)}{\tan (2 \theta) \tan (4 \theta)} \\
&=\frac{1}{\tan (2 \theta) \tan (4 \theta) \tan (8 \theta)} \sum \tan ^{3}(8 \theta) \\
&=-\frac{1}{\sqrt{7}} T(3)=31 . \\
& X Y Z=\left(\frac{\tan (4 \theta) \tan (8 \theta)}{\tan ^{2}(2 \theta)}\right)\left(\frac{\tan (8 \theta) \tan (2 \theta)}{\tan ^{2}(4 \theta)}\right)\left(\frac{\tan ^{2}(2 \theta) \tan (4 \theta)}{\tan ^{2}(8 \theta)}\right)=1 .
\end{aligned}
$$

It follows that $\{X, Y, Z\}$ are the roots of the Equation (18).

Proposition 61. For case
1) $u=1, v=1, w=1$.
2) $u=S T S 1, v=S T S 2, w=S T S 3$.
3) $u=S T S 2, v=S T S 3, w=S T S 1$.
4) $u=S T S 3, v=S T S 1, w=S T S 2$.
5) $u=S T C 1, v=S T C 2, w=S T C 3$.
6) $u=S T C 2, v=S T C 3, w=S T C 1$.
7) $u=S T C 3, v=S T C 1, w=S T C 2$.
8) $u=S T T 1, v=S T T 2, w=S T T 3$.
9) $u=S T T 2, v=S T T 3, w=S T T 1$.
10) $u=S T T 3, v=S T T 1, w=S T T 2$.

Then

$$
\left\{p(n)=u X^{n}+v Y^{n}+w Z^{n} \mid n=0,1,2, \cdots\right\}
$$

is an integer sequence with the recurrence relation

$$
p(n)=-25 p(n-1)-31 p(n-2)+p(n-3) .
$$

Proof. The proof is similar to the proof of Proposition 49.

Example 62. List of associated sequences:
1) $3,-25,563,-13297,314947,-7460905, \cdots$ [Wang A248417]
2) $0,10,-244,5790,-137176,3249666,-76983404,1823708278$,
$-43202971760, \cdots$ 


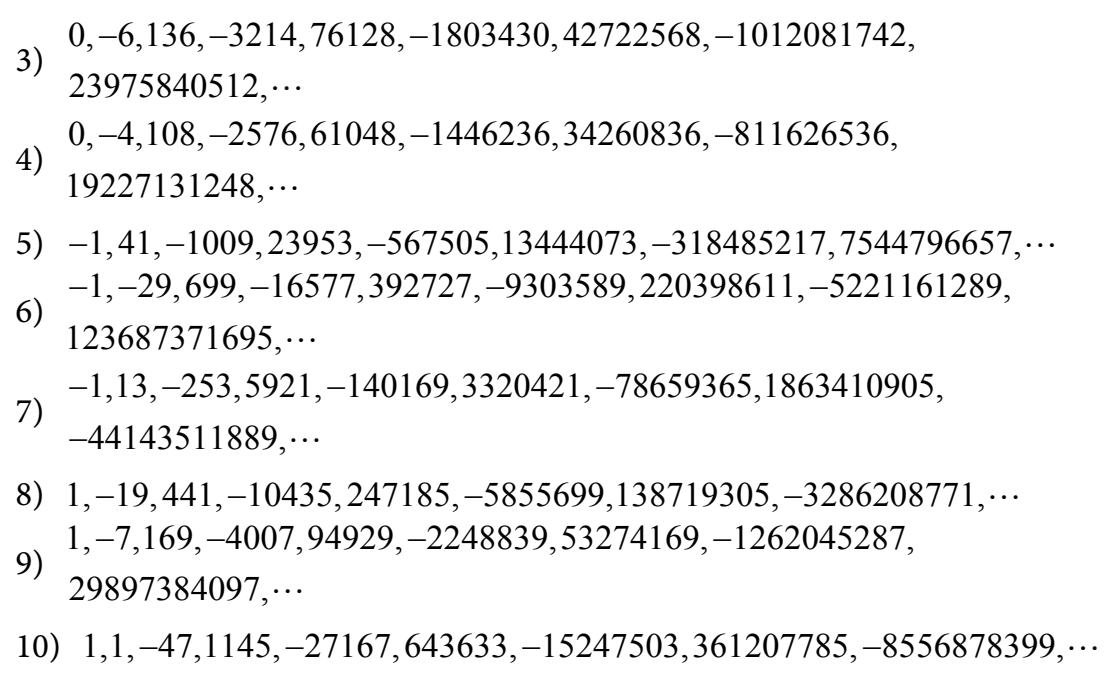

\section{Remarks}

Remark 63. All the following equations are from [3]. There are other possible cubic integer equations. For example: (A) Sine functions.

1)

$$
\begin{gathered}
x^{3}+x^{2}-9 x-1=0 . \\
X=\frac{\sin ^{3}(2 \theta)}{\sin (4 \theta) \sin ^{2}(8 \theta)}, Y=\frac{\sin ^{3}(4 \theta)}{\sin (8 \theta) \sin ^{2}(2 \theta)}, Z=\frac{\sin ^{3}(8 \theta)}{\sin (2 \theta) \sin ^{2}(4 \theta)} .
\end{gathered}
$$

2)

$$
x^{3}-5 x^{2}-8 x-1=0 \text {. }
$$

$$
X=\frac{\sin ^{3}(2 \theta)}{\sin ^{2}(4 \theta) \sin (8 \theta)}, Y=\frac{\sin ^{3}(4 \theta)}{\sin ^{2}(8 \theta) \sin (2 \theta)}, Z=\frac{\sin ^{3}(8 \theta)}{\sin ^{2}(2 \theta) \sin (4 \theta)} \text {. }
$$

3)

$$
\begin{gathered}
x^{3}-49 x+49=0 . \\
X=\frac{2 \sqrt{7} \sin ^{2}(2 \theta)}{\sin (8 \theta)}, Y=\frac{2 \sqrt{7} \sin ^{2}(4 \theta)}{\sin (2 \theta)}, Z=\frac{2 \sqrt{7} \sin ^{2}(8 \theta)}{\sin (4 \theta)} .
\end{gathered}
$$

4)

$$
\begin{gathered}
x^{3}+7 x^{2}-49 x+49=0 . \\
X=\frac{2 \sqrt{7} \sin ^{2}(2 \theta)}{\sin (4 \theta)}, Y=\frac{2 \sqrt{7} \sin ^{2}(4 \theta)}{\sin (8 \theta)}, Z=\frac{2 \sqrt{7} \sin ^{2}(8 \theta)}{\sin (2 \theta)} .
\end{gathered}
$$

5)

$$
\begin{gathered}
x^{3}-7 x+7=0 . \\
X=\frac{\sqrt{7}}{2 \sin (8 \theta)}, Y=\frac{\sqrt{7}}{2 \sin (2 \theta)}, Z=\frac{\sqrt{7}}{2 \sin (4 \theta)} .
\end{gathered}
$$

6)

$$
x^{3}-7 x^{2}+7 x+7=0 \text {. }
$$




$$
X=\frac{\sqrt{7} \sin (2 \theta)}{2 \sin ^{2}(8 \theta)}, Y=\frac{\sqrt{7} \sin (4 \theta)}{2 \sin ^{2}(2 \theta)}, Z=\frac{\sqrt{7} \sin (8 \theta)}{2 \sin ^{2}(4 \theta)} .
$$

7)

$$
\begin{gathered}
x^{3}+7 x^{2}+14 x+7=0 . \\
X=\frac{\sqrt{7} \sin (2 \theta)}{2 \sin (4 \theta) \sin (8 \theta)}, Y=\frac{\sqrt{7} \sin (4 \theta)}{2 \sin (8 \theta) \sin (2 \theta)}, Z=\frac{\sqrt{7} \sin (8 \theta)}{2 \sin (2 \theta) \sin (4 \theta)} .
\end{gathered}
$$

(B) Cosine functions:

1)

$$
x^{3}+25 x^{2}+31 x-1=0 .
$$

$$
X=\frac{\cos ^{3}(2 \theta)}{\cos (4 \theta) \cos ^{2}(8 \theta)}, Y=\frac{\cos ^{3}(4 \theta)}{\cos (8 \theta) \cos ^{2}(2 \theta)}, Z=\frac{\cos ^{3}(8 \theta)}{\cos (2 \theta) \cos ^{2}(4 \theta)} .
$$

2)

$$
\begin{gathered}
x^{3}-3 x^{2}-46 x-1=0 . \\
X=\frac{\cos ^{3}(2 \theta)}{\cos ^{2}(4 \theta) \cos (8 \theta)}, Y=\frac{\cos ^{3}(4 \theta)}{\cos ^{2}(8 \theta) \cos (2 \theta)}, Z=\frac{\cos ^{3}(8 \theta)}{\cos ^{2}(2 \theta) \cos (4 \theta)} .
\end{gathered}
$$

3)

$$
\begin{gathered}
x^{3}+8 x^{2}+5 x-1=0 . \\
X=\frac{2 \sqrt{7} \cos ^{2}(2 \theta)}{\cos (8 \theta)}, Y=\frac{2 \sqrt{7} \cos ^{2}(4 \theta)}{\cos (2 \theta)}, Z=\frac{2 \sqrt{7} \cos ^{2}(8 \theta)}{\cos (4 \theta)} .
\end{gathered}
$$

4)

$$
\begin{gathered}
x^{3}+x^{2}-9 x-1=0 . \\
X=\frac{2 \sqrt{7} \cos ^{2}(2 \theta)}{\cos (4 \theta)}, Y=\frac{2 \sqrt{7} \cos ^{2}(4 \theta)}{\cos (8 \theta)}, Z=\frac{2 \sqrt{7} \cos ^{2}(8 \theta)}{\cos (2 \theta)} .
\end{gathered}
$$

5)

$$
\begin{gathered}
x^{3}-13 x^{2}+26 x-1=0 . \\
X=\frac{2 \sqrt{7} \cos ^{3}(2 \theta)}{\cos (4 \theta) \cos (8 \theta)}, Y=\frac{2 \sqrt{7} \cos ^{3}(4 \theta)}{\cos (8 \theta) \cos (2 \theta)}, Z=\frac{2 \sqrt{7} \cos ^{3}(8 \theta)}{\cos (2 \theta) \cos (4 \theta)} .
\end{gathered}
$$

6)

$$
\begin{gathered}
x^{3}-41 x^{2}-72 x-1=0 . \\
X=\frac{2 \sqrt{7} \cos ^{4}(2 \theta)}{\cos (4 \theta) \cos ^{2}(8 \theta)}, Y=\frac{2 \sqrt{7} \cos ^{4}(4 \theta)}{\cos (8 \theta) \cos ^{2}(2 \theta)}, Z=\frac{2 \sqrt{7} \cos ^{4}(8 \theta)}{\cos (2 \theta) \cos ^{2}(4 \theta)} .
\end{gathered}
$$

7)

$$
x^{3}+22 x^{2}+103 x-1=0 .
$$

$$
X=\frac{2 \sqrt{7} \cos ^{4}(2 \theta)}{\cos ^{2}(4 \theta) \cos (8 \theta)}, Y=\frac{2 \sqrt{7} \cos ^{4}(4 \theta)}{\cos ^{2}(8 \theta) \cos (2 \theta)}, Z=\frac{2 \sqrt{7} \cos ^{4}(8 \theta)}{\cos ^{2}(2 \theta) \cos (4 \theta)} \text {. }
$$


8)

$$
\begin{gathered}
x^{3}+9 x^{2}-x-1=0 . \\
X=\frac{\cos (2 \theta)}{2 \cos ^{2}(8 \theta)}, Y=\frac{\cos (4 \theta)}{2 \cos ^{2}(2 \theta)}, Z=\frac{\cos (8 \theta)}{2 \cos ^{2}(4 \theta)} .
\end{gathered}
$$

9)

$$
\begin{gathered}
x^{3}-5 x^{2}-8 x-1=0 . \\
X=\frac{\cos (2 \theta)}{2 \cos ^{2}(4 \theta)}, Y=\frac{\cos (4 \theta)}{2 \cos ^{2}(28 \theta)}, Z=\frac{\cos (8 \theta)}{2 \cos ^{2}(2 \theta)} .
\end{gathered}
$$

10)

$$
x^{3}-5 x^{2}+6 x-1=0
$$

$$
X=\frac{\cos (2 \theta)}{2 \cos (4 \theta) \cos (8 \theta)}, Y=\frac{\cos (4 \theta)}{2 \cos (8 \theta) \cos (2 \theta)}, Z=\frac{\cos (8 \theta)}{2 \cos (2 \theta) \cos (4 \theta)} .
$$

(C) Tangent functions:

1)

$$
\begin{gathered}
x^{3}+113 x^{2}+215 x-1=0 . \\
X=\frac{\tan ^{3}(2 \theta)}{\tan (4 \theta) \tan ^{2}(8 \theta)}, Y=\frac{\tan ^{3}(4 \theta)}{\tan (8 \theta) \tan ^{2}(2 \theta)}, Z=\frac{\tan ^{3}(8 \theta)}{\tan (2 \theta) \tan ^{2}(4 \theta)} .
\end{gathered}
$$

2)

$$
\begin{gathered}
x^{3}+289 x^{2}-57 x-1=0 . \\
X=\frac{\tan ^{3}(2 \theta)}{\tan ^{2}(4 \theta) \tan (8 \theta)}, Y=\frac{\tan ^{3}(4 \theta)}{\tan ^{2}(8 \theta) \tan (2 \theta)}, Z=\frac{\tan ^{3}(8 \theta)}{\tan ^{2}(2 \theta) \tan (4 \theta)} .
\end{gathered}
$$

3)

$$
\begin{gathered}
x^{3}-49 x^{2}+343 x+49=0 . \\
X=\frac{\sqrt{7} \tan ^{2}(2 \theta)}{\tan (8 \theta)}, y=\frac{\sqrt{7} \tan ^{2}(4 \theta)}{\tan (2 \theta)}, z=\frac{\sqrt{7} \tan ^{2}(8 \theta)}{\tan (4 \theta)} .
\end{gathered}
$$

4)

$$
\begin{gathered}
x^{3}-105 x^{2}-49 x+49=0 . \\
X=\frac{\sqrt{7} \tan ^{2}(2 \theta)}{\tan (4 \theta)}, y=\frac{\sqrt{7} \tan ^{2}(4 \theta)}{\tan (8 \theta)}, z=\frac{\sqrt{7} \tan ^{2}(8 \theta)}{\tan (2 \theta)} .
\end{gathered}
$$

5)

$$
\begin{gathered}
x^{3}-7 x^{2}+7 x+7=0 . \\
X=\frac{\sqrt{7}}{\tan (8 \theta)}, Y=\frac{\sqrt{7}}{\tan (2 \theta)}, Z=\frac{\sqrt{7}}{\tan (4 \theta)} .
\end{gathered}
$$

6)

$$
x^{3}-7 x^{2}-105 x+7=0 .
$$




$$
X=\frac{\sqrt{7} \tan (2 \theta)}{\tan ^{2}(8 \theta)}, Y=\frac{\sqrt{7} \tan (4 \theta)}{\tan ^{2}(2 \theta)}, Z=\frac{\sqrt{7} \tan (8 \theta)}{\tan ^{2}(4 \theta)}
$$

7)

$$
\begin{gathered}
x^{3}+21 x^{2}+35 x+7=0 . \\
X=\frac{\sqrt{7} \tan (2 \theta)}{\tan (4 \theta) \tan (8 \theta)}, Y=\frac{\sqrt{7} \tan (4 \theta)}{\tan (8 \theta) \tan (2 \theta)}, Z=\frac{\sqrt{7} \tan (8 \theta)}{\tan (2 \theta) \tan (4 \theta)} .
\end{gathered}
$$

Remark 64. There are other possible triads. For example:
1) $\frac{2 \sin (2 \theta)}{\sqrt{7}}, \frac{2 \sin (4 \theta)}{\sqrt{7}}, \frac{2 \sin (8 \theta)}{\sqrt{7}}$.
2) $\frac{1}{4 \cos (2 \theta)}, \frac{1}{4 \cos (4 \theta)}, \frac{1}{4 \cos (8 \theta)}$.
3) $\frac{\tan (2 \theta)}{\sqrt{7}}, \frac{\tan (4 \theta)}{\sqrt{7}}, \frac{\tan (8 \theta)}{\sqrt{7}}$.

\section{Conclusions}

1) Compute all the sequences using the equations and triads in Section 19.

2) Find new methods for integer sequences.

\section{Conflicts of Interest}

The author declares no conflicts of interest regarding the publication of this paper.

\section{References}

[1] Recurrence Relation. https://en:wikipedia.org/wiki/Recurrencerelation

[2] The On-Line Encyclopedia of Integer Sequences. https://oeis:org/wiki/Welcome

[3] Wang, K. Integer Sequences for the Sum of Powers of Trigonometric Values, to Appear.

[4] Witula, R. (2009) Ramanujan Type Trigonometric Formulas: The General Form for

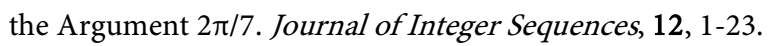

[5] Witula, R. (2010) Full Description of Ramanujan Cubic Polynomials. Journal of Integer Sequences, 13, Article 10.5.7.

[6] Witula, R. (2010) Ramanujan Cubic Polynomials of the Second Kind. Journal of Integer Sequences, 13, Article: 10.7.5.

[7] Witula, R. (2012) Ramanujan Type Trigonometric Formulae. Demonstratio Mathematica, 45, 779-796. https://doi.org/10.1515/dema-2013-0418

[8] Bankoff, L. and Garfunkel, J. (1973) The Heptagonal Triangle. Mathematics Magazine, 46, 7-19. https://doi.org/10.2307/2688574

[9] Heptagonal Triangle. https://en.wikipedia.org/wiki/Heptagonal_triangle 


\section{List of Integer Sequences}

List of integer sequences from OEIS [2] which are related to our works:

1) Adamson G. Adamson $\underline{\mathrm{A} 116423}, \underline{\mathrm{A} 180262}, \underline{\mathrm{A} 094430 .}$

2) Adamson-Bagula G. Adamson and Roger L. Bagula, A120757, A122161, $\underline{\mathrm{A} 122600 .}$.

3) Bagula R. Bagula, A106803, A152046.

4) Barry P. Barry, A094648, A096975, A096976.

5) Bernstein-Sloane-Wilson M.Bernstein, N. Sloane, R. Wilson, A000975.

6) Butler S. Butler A136776.

7) Delham P. Delham, A085810, A199853, A108716.

8) Deutsch E. Deutsch A109110.

9) encyclopedia (AT) pommard.inria.fr encyclopedia (AT) pommard.inria.fr, A052534, A052547.

10) Hanna P. Hanna, A136752.

11) Kimberling C. Kimberling, A287381.

12) W. Lang, A181879.

13) Librandi V. Librandi, $\underline{\text { A271945 }}, \underline{\text { A271944. }}$

14) Pharo A. Pharo, $\underline{A} 219788$.

15) Sloane N. Sloane, A077925, A001045, A006053, A078038, A006054, A006356, A077998, A033304, A028495.

16) Wang Kai Wang A274032, A274075, A274220, A274592, A248417, A274663, A274664, A274975, A275195, A275830, A275831, A287396, A287405, A320918.

17) Witula R. Witula, A214683, A215007, $\underline{\mathrm{A} 215008}, \underline{\mathrm{A} 215076}, \underline{\mathrm{A} 215112}$ $\underline{\mathrm{A} 215139}, \underline{\mathrm{A} 006054}, \underline{\mathrm{A} 215575}, \underline{\mathrm{A} 215100}, \underline{\mathrm{A} 215575}, \underline{\mathrm{A} 215560}, \underline{\mathrm{A} 215569}, \underline{\mathrm{A} 215666}$, A215794, A215829, A215404, A217274.

18) Wieder T. Wieder, A109509. 